\title{
Wenxin Keli for the Treatment of Arrhythmia-Systems Pharmacology and In Vivo Pharmacological Assessment
}

\section{OPEN ACCESS}

Edited by:

Xuezhong Zhou,

Beijing Jiaotong University, China

Reviewed by:

Josué Arturo Velázquez-Moyado, National Autonomous University of Mexico, Mexico

Jian Li,

Beijing University of Chinese Medicine,

China

${ }^{*}$ Correspondence:

Wuxun Du

cnduwux@163.com

Hucheng Zhao

1519411043@qq.com

${ }^{\dagger}$ These authors share first authorship

Specialty section:

This article was submitted to Ethnopharmacology,

a section of the journal

Frontiers in Pharmacology

Received: 03 May 2021

Accepted: 21 July 2021

Published: 26 August 2021

Citation:

Li X, Tian G, Xu L, Sun L, Tao R,

Zhang S, Cong Z, Deng $F$, Chen J, Yu Y, Du W and Zhao H (2021) Wenxin

Keli for the Treatment of

Arrhythmia-Systems Pharmacology

and In Vivo

Pharmacological Assessment.

Front. Pharmacol. 12:704622.

doi: 10.3389/fphar.2021.704622
Xiaofeng $\mathrm{Li}^{1+}$, Gang Tian ${ }^{2}$, Liang X Xu,4, Lili Sun ${ }^{5}$, Rui Tao ${ }^{5}$, Shaoqiang Zhang ${ }^{1}$, Zidong Cong ${ }^{1}$, Fangjun Deng ${ }^{5}$, Jinhong Chen ${ }^{5}$, Yang $\mathrm{Yu}^{6}$, Wuxun $\mathrm{Du}^{1 *}$ and Hucheng Zhao ${ }^{6 *}$

${ }^{1}$ Department of Cardiology, The Second Affiliated Hospital of Tianjin University of TCM, Tianjin, China, ${ }^{2}$ Department of Cardiology, Teda International Cardiovascular Hospital, Tianjin, China, ${ }^{3}$ School of Pharmacy, Tianjin Medical University, Tianjin, China, ${ }^{4}$ Tianjin Medical College, Tianjin, China, ${ }^{5}$ Department of TCM, Tianjin University of TCM, Tianjin, China, ${ }^{6}$ Department of Aeronautics and Astronautics, Tsinghua University, Beijing, China

This study employed a systems pharmacology approach to identify the active compounds and action mechanisms of Wenxin Keli for arrhythmia treatment. Sixty-eight components identified in vivo and in vitro by UPLC/Q-TOF-MS were considered the potential active components of Wenxin Keli. Network pharmacology further revealed 33 key targets and 75 KEGG pathways as possible pathways and targets involved in WK-mediated treatment, with the CaMKII/CNCA1C/Ca ${ }^{2+}$ pathway being the most significantly affected. This finding was validated using an AC-induced rat arrhythmias model. Pretreatment with Wenxin Keli reduced the malignant arrhythmias and shortened RR, PR, and the QT interval. Wenxin Keli exerted some antiarrhythmic effects by inhibiting $\mathrm{p}$-CaMKII and intracellular $\mathrm{Ca}^{2+}$ transients and overexpressing $\mathrm{CNCA} 1 \mathrm{C}$. Thus, suppressing $\mathrm{SR} \mathrm{Ca}^{2+}$ release and maintaining intracellular $\mathrm{Ca}^{2+}$ balance may be the primary mechanism of Wenxin Keli against arrhythmia. In view of the significance of CaMKII and NCX identified in this experiment, we suggest that CaMKII and NCX are essential targets for treating arrhythmias.

Keywords: Wenxin Keli, arrhythmia, active compounds, action mechanisms, systems phar macology, $\mathrm{Ca}^{2+}$ balance

\section{INTRODUCTION}

Arrhythmias are a group of conditions that cause the heart to beat irregularly (O’Rourke et al., 2016). It may remain asymptomatic or lead to other cardiovascular disorders, heart failure, stroke, and cardiac arrest. Severe arrhythmias, such as ventricular arrhythmias, have limited treatment options, causing them to be fatal (Hong et al., 2019). Numerous ongoing studies have reported treatment solutions for arrhythmias (Tian et al., 2019). Effective pharmaceutical agents with fewer side effects are also being explored. However,

Abbreviations: TCM, traditional Chinese medicine; WK, Wenxin Keli; Ito, potassium channel current; INa, sodium current; ICaL, L-type calcium current; APD, action potential duration; AC, aconitine; ECG, electrocardiogram; qRt-PCR, quantitative Real-time PCR; ECC, excitation-contraction coupling; LTCC, L-type $\mathrm{Ca}^{2+}$ current; AP, action potential; SR, sarcoplasmic reticulum; CACNA1C, voltage-dependent L-type calcium channel subunit alpha-1C; CaMKII, calcium/calmodulin dependent protein kinaseII; EADs, early afterdepolarizations; DADs, delayed afterdepolarization; VF, ventricular fibrillation; VT, ventricular tachycardia; VP, ventricular premature; TA, triggered activities; $\mathrm{NCX}, \mathrm{Na}^{+}-\mathrm{Ca}^{2+}$ exchanger; RyRs, ryanodine receptors; CICR, $\mathrm{Ca}^{2+}{ }_{\text {-induced }} \mathrm{Ca}^{2+}$-release; SERCA2a, $\mathrm{Ca}^{2+}$-ATPase type-2a. 
the progress is not satisfactory (Weiss et al., 2015; Camm, 2017). Though ion channels drug discovery has greatly evolved for nearly half a century, the drugs cause adverse reactions (Frommeyer and Eckardt, 2016), such as thyroid dysfunction, pulmonary fibrosis, and anaphylaxis (Salerno et al., 1990; Johannes et al., 2010; John et al., 2012). In recent years, ablation-guided electrophysiology and implantable cardioverter defibrillators have been widely used in arrhythmia treatment, achieving gratifying results (McKenna et al., 2019). Nonetheless, their promotion and use are limited by high cost, necessitating effective and cost-friendly medications.

Arrhythmias have a complex etiology and thus cannot be successfully treated using single-target therapies. As such, the multitarget treatment mode of traditional Chinese medicine (TCM) is handy in arrhythmia treatment. TCM is widely and frequently used in China and abroad (Wang et al., 2016; Liang et al., 2018). Wenxin Keli (WK) and Shensong Yangxin capsules are the most used TCM (Liang et al., 2019). Therefore, their antiarrhythmic mechanisms have been discovered (Luo et al., 2017; Zhang et al., 2017; Tian et al., 2018). WK is a Chinese herb extract comprised five components: Codonopsis pilosula (Franch.) Nannf., Panax notoginseng (Burkill) F.H.Chen, Nardostachys jatamansi (D.Don) DC., Amber, and Polygonatum kingianum Collett \& Hemsl. It is reported to be effective in arrhythmias treatment (Burashnikov et al., 2012) and is the first TCM to be approved by the Chinese state for arrhythmias management. Numerous clinical trials postulate that WK blocks the transient outward potassium channel current (Ito), sodium current (INa), and L-type calcium current (ICaL) in rat and rabbit ventricular cardiomyocytes (Liu et al., 2009; Wang et al., 2013). It also significantly shortens APD90, thus making it incompatible with the late INa blockade, which usually produces only moderate shortening of the action potential duration (APD) (Burashnikov et al., 2012). Nevertheless, the action mechanism by which WK treats arrhythmias remains unknown because the complex chemical composition and therapeutic targets of WK challenge pharmacological investigations. As such, an effective method that can decipher the relationships between the WK and arrhythmia is needed.

This study employed a systems pharmacology approach to investigate the pharmacological mechanisms of WK (Figure 1). The potential active compounds in WK were screened by ultraperformance liquid chromatography/quadrupole-time-of-flight mass spectrometry (UPLC/Q-TOF-MS), and their potential related targets were subsequently predicted using the weighted ensemble similarity method. The obtained targets were mapped onto relevant databases to detect their corresponding pathways. Subsequent experiments were further conducted to confirm whether the hypothetical results of the systemic pharmacology approach were correct (Huang et al., 2014; Li et al., 2014).

\section{MATERIALS AND METHODS}

\section{Wenxin Keli Component Identification and Prediction of Action Mechanism Component Identification}

The UPLC/Q-TOF-MS was employed to analyze aqueous solutions and serum components of WK qualitatively. The identified components were regarded as the potential active ingredients of WK.

\section{Target Prediction}

Target Proteins of WK: TCM formulas can effectively prevent the devastating effects of complex diseases through the synergistic effects of multiple compounds and targets (Hao da and Xiao, 2014). Therefore, exploring the therapeutic targets of WK is required besides identifying its potential active compounds. In this study, an integrated in silico approach was employed to identify the target proteins for the possible active ingredients (components identified from 2.1.1) of WK. Predictive models including the TCMSP database (http://ibts.hkbu.edu.hk/LSP/ tcmsp.php), STITCH (http://stitch.embl.de/) (Kuhn et al., 2012), Swiss Target Prediction (http://www. swisstargetprediction.ch/), Target-Prediction (http://prediction. charite.de/index.php?site=chemdoodle_search_target), and DrugBank (http://www.drugbank.ca/) were used to predict the target proteins of WK.

Target Proteins of Arrhythmia: Targets related to arrhythmia were obtained from "The gene connection for the heart" database (http//triad.fsm.it/cardmoc/).

\section{Network Construction}

Compound-target Network: TCM formulas exert significant biological and pharmacological effects through multiple compounds and targets. This study constructed a compoundtarget (C-T) network based on the candidate compounds of WK and the potential targets to understand the complex interaction of compounds and their corresponding targets at a systems level.

Protein-protein Interaction (PPI) Network: Networks are used to view global relationships between nodes. In this study, a PPI network was used to identify potential target proteins associated with arrhythmia based on the arrhythmia targets identified in 2.1.2 using the Cytoscape 3.8.1 Bisogenet app (Smoot et al., 2011).

\section{Identification of Key Targets}

Experimental approaches for determining the targets of candidate drugs are costly, labor-intensive, and time-consuming. In this study, a systems pharmacology approach was used to determine the key targets through which WK exerts an antiarrhythmic effect. The Merge tool of the Cytoscape 3.8.1 app was employed to determine the common targets of the C-T network and PPI network. They were regarded as the key targets by WK in arrhythmia treatment.

\section{Pathway Construction and Analysis}

Signaling pathways are essential components of the system pharmacology that link receptor-ligand interactions to pharmacodynamics outputs (Iyengar et al., 2012). An incorporated "arrhythmia pathway" was established in this study to probe the action mechanisms of WK in arrhythmia treatment based on the current knowledge of arrhythmia pathology. The pathway information was acquired by inputting the key targets from 2.1.4 into the DAVID Bioinformatics Resources 6.8 (https://david.ncifcrf.gov/). An incorporated arrhythmia pathway was then assembled based 
on the basic pathway information by picking out the closely linked pathways related to arrhythmia pathology.

\section{Experimental Procedures}

\section{Source of Reagents and Antibodies}

Acetonitrile (United States, 071757) and formic acid (analytical grade) were purchased from Fisher Scientific (United States, 154449). Purified water was acquired from a Milli-Q system (Millipore, Bedford, MA, United States). Medlab RU/4C50R multi-channel physiological signal acquisition and processing system were purchased from Shanghai Tongyu Teaching Instrument Manufacturing Co., Ltd (batch No. 17060155). WK was purchased from Shandong Buchang Pharmaceutical Co., Ltd, Tianjin, China (SFDA Approval number 1805106). Aconitine (AC) was sourced from Baoji Chenguang Biotechnology Co., Ltd., Shanxi, China (batch No. ha001198). Pentobarbital sodium was purchased from Baoji Chenguang Biotechnology Co., Ltd., Shanxi, China (batch No. B21882). In the same line, the p-CaMKII rabbit antibody was sourced from Abcam (ab32678), the GAPDH rabbit antibody from Hangzhou Xianzhi biology Co., Ltd, China (AB-P-R 001), and the HRP labeled Goat anti-rabbit second antibody from Wuhan bode Bioengineering Co., Ltd, China (BA1054). The cDNA synthesis kit (K1622) was purchased from ThermoFisher Scientific, United States.

\section{Sample Preparation}

WK (5 g) was dissolved in $20.6 \mathrm{ml}$ purified water (Nongfu Spring) and left to stand for $1 \mathrm{~h}$. The solution was then filtered through four layers of gauze to filter out the dregs. The filtrate was subsequently centrifuged for $10 \mathrm{~min}$ at $10,000 \mathrm{rpm}$ and $4^{\circ} \mathrm{C}$, and $300 \mathrm{ml}$ of the supernatant was stored at $-20^{\circ} \mathrm{C}$. The supernatant was diluted four times and filtered through a $0.22 \mu \mathrm{m}$ filter membrane before injection for UPLC/Q-TOFMS determination.

Blood samples $(0.5 \mathrm{ml})$ were collected into serum tubes from the eye sockets of rats after $0.5,1,1.5$, and $2 \mathrm{~h}$ of WK administration followed by immediate centrifugation at $3,500 \mathrm{rpm}$ for $15 \mathrm{~min}$ at $4 \mathrm{C}$. The extracted serum was then subjected to UPLC/Q-TOF-MS determination.

\section{Instrumentation and Chromatographic Conditions}

Chromatographic separation was performed on an ACQUITY UPLC BEH C ${ }_{18}$ column $(\Phi 2.1 \mathrm{~mm} \times 100 \mathrm{~mm}, 1.7 \mu \mathrm{m}$; Waters, United States) at a column temperature of $45^{\circ} \mathrm{C}$ and $5 \mu$ injection volume. The sample was eluted at a flow rate of $0.3 \mathrm{ml} \mathrm{min}^{-1}$ in a gradient elution program of $\mathrm{A}(0.1 \%$ formic acid: water $)$ and $\mathrm{B}$ ( $0.1 \%$ acetonitrile: water): $0-6 \mathrm{~min}(98-78 \% \mathrm{~A}) ; 6-10 \mathrm{~min}$ (78-70\% A); $10-14 \min (70-62 \% \mathrm{~A}) ; 14-18 \min (62-62 \% \mathrm{~A})$ $18-22 \min (62-40 \%$ A); $22-26 \min (40-0 \%$ A); $26-28 \min (0-0 \%$ A); 28-29 min (0-98\% A) 29-30 min (98-98\% A). MS detection was performed on a high definition MS (HDMS) system (Waters, Te United States) with negative and positive electrospray (ESI) modes. Its sufficient sensitivity ensured that as many putative compounds as possible were identified. The optimized operating parameters were capillary voltage; $3.0 \mathrm{kV}$ (Positive)/ $2.0 \mathrm{kV}$ (Negative), source temperature; $120^{\circ} \mathrm{C}$ (Positive) $/ 110^{\circ} \mathrm{C}$
(Negative), desolvation gas temperature; $450^{\circ} \mathrm{C}$, desolvation gas $\left(\mathrm{N}_{2}\right)$ flow rate; $800 \mathrm{~L} / \mathrm{h}$, collision energy $(\mathrm{CE}) ; 25 \mathrm{~V}$, scanning time; $0.1 \mathrm{~s}$, and scanning time interval; $0.02 \mathrm{~s}$. Leucine-cerebral peptide solution (Waters) at a concentration of $200 \mathrm{pg} / \mathrm{ml}$ was used as the Lock-Spray calibration solution to ensure the accuracy and repeatability of the mass-to-charge ratio. The exact mass-tocharge ratios in positive and negative ion modes were $[\mathrm{M}+\mathrm{H}]^{-}=$ 556.2771 and $[\mathrm{M}-\mathrm{H}]^{-}=554.2615$, respectively. The data acquisition range and time were $\mathrm{m} / \mathrm{z} 50-1000$ and $0-30 \mathrm{~min}$, respectively.

\section{Animals Used and the Experimental Design}

Clean grade male Sprague Dawley (SD, SPF level) rats aged 6-7 weeks and weighing $220 \pm 20 \mathrm{~g}$ were obtained from the Experimental Animal Center of the Institute of health and environmental medicine, Academy of Military Medical Sciences, PLA, [license no. SYXK(Jin) 2014-0002]. All the animal-based experiments were approved by the Animal Care Committee of the Institute of Radiation Medicine of the Chinese Academy of Medical Sciences and performed following the relevant guidelines and regulations. Efforts were also made to minimize animal suffering. All rats were housed in the specific pathogen-free (SPF) animal laboratory of the experimental animal center of the Institute of Radiation Medicine Chinese Academy of Medical Sciences at a temperature of $23^{\circ} \mathrm{C} \pm 2^{\circ} \mathrm{C}$ and $35 \% \pm 5 \%$ relative humidity. The animals were acclimatized to the environment for 1 week with free access to a standard pellet diet before initiating the experiments. The rats were randomly divided into six groups, each comprising eight animals: the control (C) group, model (M) group, low-dose WK-treated (LD) group, medium-dose WK-treated (MD) group, high-dose WK-treated (HD) group, and the amiodarone (A) group. Intragastric administration of WK was once a day for 14 consecutive days in groups LD $(2.41 \mathrm{~g} / \mathrm{kg} / \mathrm{d}), \quad \mathrm{MD}(4.82 \mathrm{~g} / \mathrm{kg} / \mathrm{d})$, and $\mathrm{HD}$ $(9.64 \mathrm{~g} / \mathrm{kg} / \mathrm{d})$ (values are for the crude drug). Rats in group A were administrated with amiodarone $(40 \mathrm{mg} / \mathrm{kg})$, while those in groups $\mathrm{C}$ and $\mathrm{M}$ were administrated with an equal volume of distilled water.

\section{Electrocardiogram Analysis}

The rats were intraperitoneally anesthetized with $0.6 \%$ pentobarbital $30 \mathrm{mg} / \mathrm{kg}$ an hour after the last WK administration. They were then fixed on the plank in a prone position, followed by the insertion of electrodes into the subcutaneous tissue of the limb. The ECG parameters were subsequently recorded in each group using a Medlab RU/ 4C50R multi-channel physiological signal acquisition and processing system via a standard limb lead II. Rats in the other five groups, except those in group $\mathrm{C}$, were subsequently injected with $0.001 \%$ AC $40 \mu \mathrm{g} / \mathrm{kg}$ after ECG stabilization to establish the arrhythmia model. The injection was through the caudal vein at a rate of $0.2 \mathrm{ml} / \mathrm{min}$. Equal volumes of $0.9 \%$ normal saline were injected into rats in group C. The ECG of the rats was then observed in real-time for $20 \mathrm{~min}$ after the heart rhythm had normalized. The parameters measured included the initial time of arrhythmia and the types, occurrence, and duration of various VAs. 
All animals were euthanized after ECG detection, and their hearts were immediately harvested and stored in liquid nitrogen awaiting WB and qRt-PCR analysis.

\section{Western Blotting Analysis}

The proteins were separated on a $10 \%$ SDS-PAGE and then transferred onto nitrocellulose membranes. The membranes were subsequently incubated with p-CaMKII rabbit antibody at $4^{\circ} \mathrm{C}$ overnight and then washed thrice with Tris-buffered saline (TBS) containing Tween 20 (TBST) to wash off the excess antibodies. The membranes were further incubated with horseradish peroxidase-conjugated secondary antibody for $2 \mathrm{~h}$ at room temperature. ECL visualization was then performed, followed by calculating the gray values using the Image J software (NIH Image, Bethesda, MD, United States). The intensity of the target proteins was normalized to that of an internal reference to determine their relative expression level.

\section{Quantitative Real-Time PCR Analysis}

The mRNA expression level of the predicated WK CAMKII/ $\mathrm{CNCA} 1 \mathrm{C} / \mathrm{Ca}^{2+}$ pathway was validated using Real-time Quantitative PCR (RT-qPCR) analysis. The total RNA of the myocardial pieces was first isolated using the Trizol reagent (Ambion, 15596-026), following the manufacturer's instructions. The RNA samples $(5 \mu \mathrm{g})$ were then reversetranscribed to complementary DNA (cDNA) using a cDNA synthesis kit, followed by quantitative Rt-PCR on a 7500 Fast Real-Time PCR System (Applied Biosystems). The primer pair used was CAMKII forward; 5'-CAAGTTCATCGAGGTCAC CAC- $3^{\prime}$ and reverse; 5'-ATACACAGCTCTCGTCCTCTG- ${ }^{\prime}$, with RNA U6 as the internal control. The miR-1 specific primer sequences for quantitative real-time PCR were used for TaqMan MicroRNA Assays (Catalog number 4427975, Applied Biosystems). The expression levels of the miR-1 were subsequently normalized to U6 RNA expression levels and then calculated using the $2-\Delta \Delta \mathrm{Ct}$ method (Livak and Schmittgen, 2001).

\section{Laser-Scanning Confocal Calcium Imaging}

Calcium imaging was carried out at room temperature within $6 \mathrm{~h}$ after isolation of cardiomyocytes from rats in the control group (Zhang et al., 2020). $\mathrm{Ca}^{2+}$ transients in the cardiomyocytes were recorded using an LSM-710 laser-scanning confocal microscope (Carl Zeiss, Inc, Germany) with a $\times 40$ magnification, 1.3 numerical aperture oil immersion objective, and axial resolutions of $1.5 \mu \mathrm{m}$. Fluo- 4 AM: Fluo- $4 \mathrm{AM}+8 \mu \mathrm{lDMSO}+2 \mu \mathrm{lF}-$ 127 was first prepared and then diluted 1,000 times using $1 \mathrm{ml}$ $\mathrm{DMem}+1 \mu \mathrm{l}$ Fluo-4. Cardiomyocytes were then incubated with 500-10,002 $\mu$ l Fluo-4 AM (AAT Bioquest, Inc. Sunnyvale, CA, United States) for $20 \mathrm{~min}$ at $37^{\circ} \mathrm{C}$ and recorded in normal Tyrode's solution. Fluo- 4 was excited at $488 \mathrm{~nm}$, followed by measurement of the fluorescence emission at $505 \mathrm{~nm}$. Images were acquired in the line-scan (X-T) mode with 512 pixels (pixel intervals $0.15 \mu \mathrm{m}$ ) per line at a rate of $3 \mathrm{~ms}$ per scan. The $\mathrm{Ca}^{2+}$ transients were then analyzed using a modified version of the MATLAB program, and their fluorescence emission intensity was expressed as F/F0, where F0 was the basal fluorescence intensity level. The recording was performed at $35^{\circ} \mathrm{C}$.

\section{Statistical Analysis}

Data were expressed as the means \pm SEM. Differences between the two groups were analyzed using the Student's two-tailed $t$-test, while those between multiple groups were analyzed using one-way analysis of variance (SPSS, Inc., Chicago, IL, United States). $p<0.05$ indicated significant differences between groups.

\section{RESULTS}

\section{Screening of Potential Active Compounds}

UPLC/Q-TOF-MS detected 51 components from the WK aqueous solution based on the $\mathrm{m} / \mathrm{z}$ of precursors and fragments. Sugars and saponins were the main constituents of the components. The serum obtained after WK treatment contained 25 compounds, mainly composed of sugars, saponins, flavonoids, and amino acids. There were eight common components between the two solutions, and other 68 components were identified in vitro and in vivo, which were regarded as the potential active components in this study (Tables 1 and 2; Figures 2, 3).

\section{Target Prediction}

Target proteins of WK: There were 192 potential targets that were predicted for the 68 candidate compounds. However, 34 candidate compounds had no corresponding targets based on this method (Supplementary Appendix S1). The five herbs had significant target overlap among them despite the differences in the number of each herb-related target. These results suggested that the different herbal drugs contained in WK possibly worked synergistically in regulating similar targets.

Target Proteins of Arrhythmia: The gene connection for the heart (http//triad.fsm.it/cardmoc/) revealed 61 targets related to arrhythmia (Supplementary Appendix S2).

\section{Network Construction}

Compound-target network: The C-T network embodied 226 nodes (34 candidate compounds and 192 potential targets) and 429 compound-target interactions. The mean degree value (the number of targets associated with it) of the candidate compounds was 41. Twelve compounds possessed a degree larger than 14 (2-fold degree median), indicating that they regulated multiple targets to exert varying therapeutic effects. Specially, five compounds, including Atractylenolide II, Tanshinone IIA, ginsenoside Rh2, Ginsenoside Rg1, and Jatamansinol, acted on 28, 24, 21, 20, and 16 targets, respectively. The compounds were regarded as the crucial active compounds for WK because of their prime positions in the network.

PPI network of arrhythmia: There were 1395 targets closely related to known arrhythmia targets. They were regarded to be indirectly related to arrhythmia. 
TABLE 1 | Main components of WK aqueous solution.

\begin{tabular}{|c|c|c|c|c|c|c|c|c|}
\hline No. & $\mathrm{tR} / \mathrm{min}$ & Nane & Formula & $\begin{array}{l}\text { Calculated } \\
\text { mass }\end{array}$ & $\begin{array}{l}\text { Measured } \\
\text { mass }\end{array}$ & $\begin{array}{l}\text { Error } \\
\text { (ppm) }\end{array}$ & $\begin{array}{c}\text { MS/MS } \\
\text { fragmentation }\end{array}$ & Herb \\
\hline 1 & 0.74 & GUP & $\mathrm{C} 6 \mathrm{H} 12 \mathrm{O} 6$ & 180.156 & 180.063 & 6.201117318 & $\begin{array}{c}179.0567[\mathrm{M}-\mathrm{H}]- \\
225.0627[\mathrm{M}+\mathrm{CHOO}]-\end{array}$ & $1,2,3,4$ \\
\hline 2 & 0.77 & D-(+)-Xylose & $\mathrm{C} 5 \mathrm{H} 10 \mathrm{O} 5$ & 150.13 & 150.053 & 7.692307692 & 195.0520[M+CHOO]- & 3 \\
\hline 3 & 1.18 & Jatamansinol & $\mathrm{C} 14 \mathrm{H} 14 \mathrm{O} 4$ & 246.262 & 246.089 & 9.931271478 & 291.0898[M+CHOO]- & 3 \\
\hline 4 & 1.28 & Oroselol & $\mathrm{C} 14 \mathrm{H} 12 \mathrm{O} 4$ & 244.246 & 244.074 & 6.049382716 & 243.0643[M-H]- & 3 \\
\hline 5 & 1.46 & Succinic acid & $\mathrm{C} 4 \mathrm{H} 6 \mathrm{O} 4$ & 118.088 & 118.027 & 6.923076923 & $\begin{array}{c}117.0196[\mathrm{M}-\mathrm{H}]- \\
99.0118[\mathrm{M}-\mathrm{H}-\mathrm{H} 2 \mathrm{O}]- \\
73.0313[\mathrm{M}-\mathrm{H}-\mathrm{CO} 2]-\end{array}$ & 1,4 \\
\hline 6 & 1.75 & Leucine & $\mathrm{C} 6 \mathrm{H} 13 \mathrm{NO} 2$ & 131.175 & 131.095 & 9.615384615 & 130.0881[M-H]- & 4 \\
\hline 7 & 1.97 & n-Butyl- $\beta$-D-fructopyranoside & $\mathrm{C} 10 \mathrm{H} 2006$ & 236.264 & 236.126 & 9.928825623 & $\begin{array}{c}281.1265[\mathrm{M}+\mathrm{CHOO}]- \\
341.1112 \\
{[\mathrm{M}+2 \mathrm{CHOO}+\mathrm{H} 2 \mathrm{O}-3 \mathrm{H}]-}\end{array}$ & 4 \\
\hline 8 & 2.03 & Salicylic acid & $\mathrm{C} 7 \mathrm{H} 6 \mathrm{O} 3$ & 138.122 & 138.032 & 5.255474453 & $137.0246[\mathrm{M}-\mathrm{H}]-$ & 4 \\
\hline 9 & 2.51 & Codonopsine & $\mathrm{C} 14 \mathrm{H} 21 \mathrm{NO} 4$ & 267.325 & 267.147 & 3.246268657 & $268.1541[\mathrm{M}+\mathrm{H}]+$ & 1 \\
\hline 10 & 2.85 & 5-Methoxymethyl furfural & $\mathrm{C} 7 \mathrm{H} 8 \mathrm{O} 3$ & 140.138 & 140.047 & 8.957055215 & 163.0386[M+Na]+ & 1 \\
\hline 11 & 2.97 & a-Copaene & $\mathrm{C} 15 \mathrm{H} 24$ & 204.357 & 204.188 & 4.36123348 & $\begin{array}{l}249.1600[\mathrm{M}+\mathrm{CHOO}]- \\
227.1767[\mathrm{M}+\mathrm{Na}]+\end{array}$ & 2 \\
\hline 12 & 3.88 & Chlorogenic acid & $\mathrm{C} 16 \mathrm{H} 18 \mathrm{O} 9$ & 354.311 & 354.095 & 4.532577904 & 353.0889[M-H]- & 3 \\
\hline 13 & 4.95 & Cryptotanshinone (1) & $\mathrm{C} 19 \mathrm{H} 2003$ & 296.366 & 296.141 & 2.033898305 & 295.1335[M-H]- & 3 \\
\hline 14 & 4.97 & Tanshinone $\| \mathrm{A}$ & $\mathrm{C} 19 \mathrm{H} 18 \mathrm{O} 3$ & 294.35 & 294.126 & 6.962457338 & $\begin{array}{l}293.1158[\mathrm{M}-\mathrm{H}]- \\
295.1329[\mathrm{M}+\mathrm{H}]+\end{array}$ & 3 \\
\hline 15 & 5.03 & Tangshenoside III (2) & $\mathrm{C} 34 \mathrm{H} 46 \mathrm{O} 17$ & 726.725 & 726.274 & 4.409857328 & $771.2679[\mathrm{M}+\mathrm{CHOO}]-$ & 1 \\
\hline 16 & 5.07 & $\begin{array}{l}(+) \text {-Pinoresinol-O- } \beta \text {-D-glucopyranosyl }(1 \rightarrow 6) \text { - } \\
\beta \text {-D-glucopyranoside }\end{array}$ & $\mathrm{C} 32 \mathrm{H} 42 \mathrm{O} 16$ & 682.672 & 682.247 & 1.600587371 & 681.2385[M-H]- & 4 \\
\hline 17 & 5.82 & 3,5-Dimethoxyacetophenone & $\mathrm{C} 10 \mathrm{H} 12 \mathrm{O} 3$ & 180.203 & 180.079 & 7.29281768 & 181.0852[M-H]- & 2 \\
\hline 18 & 5.98 & $\begin{array}{l}\text { (1R)-2,3,4,9-tetrahydro-1H-\$b-carboline-1- } \\
\text { carboxylic acid }\end{array}$ & $\mathrm{C} 12 \mathrm{H} 12 \mathrm{~N} 2 \mathrm{O} 2$ & 216.24 & 216.09 & 5.760368664 & $217.0965[\mathrm{M}+\mathrm{H}]+$ & 1 \\
\hline 19 & 6.14 & Heptanoic acid & $\mathrm{C} 7 \mathrm{H} 14 \mathrm{O} 2$ & 130.187 & 130.099 & 4.057142857 & $175.0978[\mathrm{M}+\mathrm{CHOO}]-$ & 1 \\
\hline 20 & 6.16 & $\begin{array}{l}\text { Hexyl- } \beta \text {-D-glucopyranosyl-( }(1 \rightarrow 2)-\beta-D \text { - } \\
\text { glucopyranoside }\end{array}$ & $\mathrm{C} 18 \mathrm{H} 34011$ & 426.459 & 426.21 & 3.529411765 & 425.2039[M-H]- & 1 \\
\hline 21 & 6.72 & Kingianoside A & С39H60014 & 752.895 & 752.398 & 0.836653386 & $753.407[\mathrm{M}+\mathrm{H}]+$ & 4 \\
\hline 22 & 7.15 & Azelaic acid & $\mathrm{C} 9 \mathrm{H} 16 \mathrm{O} 4$ & 188.223 & 188.105 & 3.262032086 & $\begin{array}{c}187.0977[\mathrm{M}-\mathrm{H}]- \\
125.0979[\mathrm{M}-\mathrm{H}- \\
\mathrm{H} 2 \mathrm{O}-\mathrm{CO} 2]-\end{array}$ & 1 \\
\hline 23 & 7.19 & 20-O-Glucopyranosyl ginsenoside Rf & $\mathrm{C} 48 \mathrm{H} 82 \mathrm{O} 19$ & 963.165 & 962.545 & 0.793650794 & $\begin{array}{l}1007.5438[\mathrm{M}+\mathrm{CHOO}]- \\
961[\mathrm{M}-\mathrm{H}]-\end{array}$ & 2 \\
\hline 24 & 7.33 & Myristicin & $\mathrm{C} 11 \mathrm{H} 12 \mathrm{O} 3$ & 192.214 & 192.079 & 0.932642487 & 193.0867[M+H]+ & 1 \\
\hline 25 & 7.36 & Notoginsenoside $\mathrm{Fe}$ (3) & $\mathrm{C} 47 \mathrm{H} 80017$ & 917.14 & 916.54 & 1.746361746 & 961.5392[M+CHOO]- & 2 \\
\hline 26 & 7.65 & Gynunol & $\mathrm{C} 11 \mathrm{H} 14 \mathrm{O} 3$ & 194.23 & 194.094 & 2.510460251 & $239.0926[\mathrm{M}+\mathrm{Na}]+$ & 2 \\
\hline 27 & 7.78 & Notoginsenoside R3 & $\mathrm{C} 48 \mathrm{H} 82 \mathrm{O} 19$ & 963.165 & 962.545 & 0.793650794 & $1007.5439[\mathrm{M}+\mathrm{CHOO}]-$ & 2 \\
\hline 28 & 7.83 & Lobetyolin & $\mathrm{C} 2 \mathrm{OH} 28 \mathrm{O} 8$ & 396.436 & 396.178 & & 419.1693[M+Na]+ & 1 \\
\hline 29 & 8.02 & Notoginsenoside R1 & $\mathrm{C} 47 \mathrm{H} 80018$ & 933.139 & 932.534 & 0.27607362 & $\begin{array}{l}977.5327[\mathrm{M}+\mathrm{CHOO}]- \\
931.5277[\mathrm{M}-\mathrm{H}]-\end{array}$ & 1,2 \\
\hline 30 & 8.56 & ginsenoside Rh2 & $\mathrm{C} 36 \mathrm{H} 62 \mathrm{O} 8$ & 622.884 & 622.444 & 8.780096308 & 623.4471[M+H]+ & 2 \\
\hline 31 & 8.58 & a-Curcumene & $\mathrm{C} 15 \mathrm{H} 22$ & 202.341 & 202.172 & 5.960591133 & 203.1813[M+H\}+ & 1 \\
\hline 32 & 8.59 & Ginsenoside Rg1 & $\mathrm{C} 42 \mathrm{H} 72 \mathrm{O} 14$ & 801.024 & 800.492 & 0.059171598 & $\begin{array}{l}845.4901[\mathrm{M}+\mathrm{CHOO}]- \\
799.4858[\mathrm{M}-\mathrm{H}]-\end{array}$ & 2 \\
\hline 33 & 8.65 & Dioscin & $\mathrm{C} 45 \mathrm{H} 72 \mathrm{O} 16$ & 869.055 & 868.482 & 2.595837897 & $913.4776[\mathrm{M}+\mathrm{CHOO}]-$ & 4 \\
\hline 34 & 10.52 & Panaxytriol (4) & $\mathrm{C} 17 \mathrm{H} 26 \mathrm{O} 3$ & 278.392 & 278.188 & 5.519713262 & 279.1946[M+H]+ & 2 \\
\hline 35 & 10.92 & Notoginsenoside R6 & $\mathrm{C} 48 \mathrm{H} 82 \mathrm{O} 19$ & 963.165 & 962.545 & 0.793650794 & $\begin{array}{c}\text { 1007.5438[M+CHOO }]- \\
843.4697 \\
{[\mathrm{M}+\mathrm{CHOO}-\mathrm{Rha}]-}\end{array}$ & 2 \\
\hline 36 & 11.3 & Notoginsenoside $T$ & $\mathrm{C} 64 \mathrm{H} 108 \mathrm{O} 31$ & 1373.537 & 1372.687 & 1.909620991 & $1371.6774[\mathrm{M}-\mathrm{H}]-$ & 2 \\
\hline 37 & 11.82 & Notoginsenoside $\mathrm{Fa}$ & $\mathrm{C} 59 \mathrm{H} 100 \mathrm{O} 27$ & 1241.422 & 1240.645 & 2.532258064 & $\begin{array}{c}\text { 1239.6346[M-H]- } \\
1285.6412[\mathrm{M}+\mathrm{CHOO}]-\end{array}$ & 2 \\
\hline 38 & 12.32 & Notoginsenoside R4 & $\mathrm{C} 59 \mathrm{H} 100 \mathrm{O} 27$ & 1241.422 & 1240.645 & 3.177419355 & $\begin{array}{c}1239.6338[\mathrm{M}-\mathrm{H}]- \\
1285.6387[\mathrm{M}+\mathrm{CHOO}]-\end{array}$ & 2 \\
\hline 39 & 12.42 & Majonoside R1 & $\mathrm{C} 42 \mathrm{H} 72 \mathrm{O} 15$ & 817.023 & 816.487 & 0.392638037 & $\begin{array}{c}\text { 815.4799[M-H]- } \\
861.4844[\mathrm{M}+\mathrm{CHOO}]-\end{array}$ & 2 \\
\hline 40 & 12.51 & Dehydroadynerigenin digitaloside & $\mathrm{C} 30 \mathrm{H} 42 \mathrm{O} 8$ & 530.658 & 530.288 & 4.365217391 & $575.2883[\mathrm{M}+\mathrm{CHOO}]-$ & 4 \\
\hline 41 & 12.9 & Ginsenoside Rb1 & $\mathrm{C} 54 \mathrm{H} 92 \mathrm{O} 23$ & 1109.307 & 1108.603 & 0.23465704 & $1107.5952[\mathrm{M}-\mathrm{H}]-$ & 2 \\
\hline 42 & 12.99 & Ginsenoside Rg3 & $\mathrm{C} 42 \mathrm{H} 72 \mathrm{O} 13$ & 785.025 & 784.497 & 0.434258142 & $\begin{array}{c}\text { 829.4956[M+CHOO]- } \\
783.4899[\mathrm{M}-\mathrm{H}]- \\
\text { (Continued on followi }\end{array}$ & $\begin{array}{r}2 \\
\text { page) }\end{array}$ \\
\hline
\end{tabular}


TABLE 1 | (Continued) Main components of WK aqueous solution.

\begin{tabular}{|c|c|c|c|c|c|c|c|c|}
\hline No. & $\mathrm{tR} / \mathrm{min}$ & Nane & Formula & $\begin{array}{l}\text { Calculated } \\
\text { mass }\end{array}$ & $\begin{array}{l}\text { Measured } \\
\text { mass }\end{array}$ & $\begin{array}{l}\text { Error } \\
\text { (ppm) }\end{array}$ & $\begin{array}{c}\text { MS/MS } \\
\text { fragmentation }\end{array}$ & Herb \\
\hline 43 & 13.08 & Ginsenoside Rh1 & C36H62O9 & 638.883 & 638.439 & 1.171303075 & 683.4381[M+CHOO]- & 2 \\
\hline 44 & 13.38 & Notoginsenoside Fc & $\mathrm{C} 58 \mathrm{H} 98 \mathrm{O} 26$ & 1211.396 & 1210.635 & 2.537190083 & 1209.6241[M-H]- & 2 \\
\hline 45 & 13.85 & Ginsenoside Rb2 & C53H90O22 & 1079.281 & 1078.592 & 0.204626335 & $\begin{array}{c}1123.5906[\mathrm{M}+\mathrm{CHOO}]- \\
1077.5852[\mathrm{M}-\mathrm{H}]-\end{array}$ & 2 \\
\hline 46 & 14.48 & Sanchinoside B1 (5) & C36H62O9 & 638.883 & 638.439 & 1.171303075 & 683.4393[M+CHOO]- & 2 \\
\hline 47 & 14.94 & Ginsenoside Rd & $\mathrm{C} 48 \mathrm{H} 82 \mathrm{O} 18$ & 947.166 & 946.55 & 1.199596774 & $\begin{array}{c}991.5469[\mathrm{M}+\mathrm{CHOO}]- \\
945.5406[\mathrm{M}-\mathrm{H}]-\end{array}$ & 2 \\
\hline 48 & 15.97 & Ginsenoside Re & $\mathrm{C} 48 \mathrm{H} 82 \mathrm{O} 18$ & 947.166 & 946.55 & 1.199596774 & $\begin{array}{c}991.5469[\mathrm{M}+\mathrm{CHOO}]- \\
945.5410[\mathrm{M}-\mathrm{H}]-\end{array}$ & 2 \\
\hline 49 & 20.01 & Ginsenoside Rh4 (6) & $\mathrm{C} 36 \mathrm{H} 6008$ & 620.868 & 620.429 & 3.654135338 & $665.4254[\mathrm{M}+\mathrm{CHOO}]-$ & 2 \\
\hline 50 & 22.58 & Atractylenolide // (7) & $\mathrm{C} 15 \mathrm{H} 20 \mathrm{O} 2$ & 232.323 & 232.146 & 1.335740072 & 277.1437[M+CHOO]- & 1 \\
\hline 51 & 24.14 & Ditertbutyl phthalate (8) & $\mathrm{C} 16 \mathrm{H} 22 \mathrm{O} 4$ & 278.348 & 278.152 & 4.418604651 & $\begin{array}{c}277.1437[\mathrm{M}-\mathrm{H}]- \\
323.1353[\mathrm{M}+\mathrm{CHO} 2]-\end{array}$ & 2 \\
\hline
\end{tabular}

a1: Codonopsis pilosula (Franch.) Nannf.; 2: Panax notoginseng (Burkill) F.H.Chen; 3: Nardostachys jatamansi (D.Don) DC.; 4: Polygonatum kingianum Collett \& Hemsl. The eight components in italic are common components in vivo and in vitro. Reference: Wang and Qian (2010).

TABLE 2 | Main components of serum after treatment of WK.

\begin{tabular}{|c|c|c|c|c|c|c|c|c|}
\hline No. & $\mathrm{tR} / \mathrm{min}$ & Nane & Formula & $\begin{array}{l}\text { Calculated } \\
\text { mass }\end{array}$ & $\begin{array}{l}\text { Measured } \\
\text { mass }\end{array}$ & $\begin{array}{l}\text { Error } \\
\text { (ppm) }\end{array}$ & $\begin{array}{c}\text { MS/MS } \\
\text { fragmentation }\end{array}$ & Herb \\
\hline 1 & 0.84 & FOA & $\mathrm{C} 5 \mathrm{H} 4 \mathrm{O} 3$ & 112.084 & 112.016 & 0.888888889 & $135.0057[\mathrm{M}+\mathrm{Na}]+$ & 1 \\
\hline 2 & 0.85 & Debilone & $\mathrm{C} 15 \mathrm{H} 22 \mathrm{O} 2$ & 234.339 & 234.162 & 1.361867704 & $257.1522[\mathrm{M}+\mathrm{Na}]+$ & 3 \\
\hline 3 & 1.03 & Ricinin & $\mathrm{C} 8 \mathrm{H} 8 \mathrm{~N} 2 \mathrm{O} 2$ & 164.164 & 164.059 & 1.636363636 & $165.0667[\mathrm{M}+\mathrm{H}]+$ & 1 \\
\hline 4 & 4.31 & Oroxin A & $\mathrm{C} 21 \mathrm{H} 20010$ & 432.381 & 432.106 & 3.76443418 & 433.1119[M+H]+ & 4 \\
\hline 5 & 7.08 & Picein & $\mathrm{C} 14 \mathrm{H} 18 \mathrm{O} 7$ & 298.291 & 298.105 & 7.138047138 & 297.0996[M-H]- & 2 \\
\hline 6 & 16.26 & Isophytol & $\mathrm{C} 2 \mathrm{OH} 40 \mathrm{O}$ & 296.539 & 296.308 & 3.510971787 & $319.299[\mathrm{M}+\mathrm{Na}]+$ & 3 \\
\hline 7 & 21.79 & Panaxytriol (4) & $\mathrm{C} 17 \mathrm{H} 26 \mathrm{O} 3$ & 278.392 & 278.188 & 3.176895307 & 277.1796[M-H]- & 2 \\
\hline 8 & 22.4 & Atractylenolide // (7) & $\mathrm{C} 15 \mathrm{H} 20 \mathrm{O} 2$ & 232.323 & 232.146 & 0.83032491 & $\begin{array}{c}277.1443 \\
{[\mathrm{M}+\mathrm{CHOO}]-}\end{array}$ & 1 \\
\hline 9 & 23.21 & Majonoside R2 & $\mathrm{C} 41 \mathrm{H} 70014$ & 786.997 & 786.477 & 3.226205191 & $809.464[\mathrm{M}+\mathrm{Na}]+$ & 2 \\
\hline 10 & 24.6 & Ditertbutyl phthalate (8) & $\mathrm{C} 16 \mathrm{H} 22 \mathrm{O} 4$ & 278.348 & 278.152 & 0.431893688 & $\begin{array}{c}277.1443[\mathrm{M}-\mathrm{H}]- \\
301.1418[\mathrm{M}+\mathrm{Na}]+\end{array}$ & 2 \\
\hline 11 & 25.79 & $\begin{array}{l}\text { 1-Methyl-4-isoallyl- } \\
\text { cyclohexane }\end{array}$ & $\mathrm{C} 10 \mathrm{H} 18 \mathrm{O} 2$ & 126.111 & 126.032 & 4.152046784 & $171.1393[\mathrm{M}+\mathrm{H}]+$ & 2 \\
\hline 12 & 25.86 & Notoginsenoside Fe (3) & $\mathrm{C} 47 \mathrm{H} 80017$ & 917.14 & 916.54 & 2.68558952 & 915.5345[M-H]- & 2 \\
\hline 13 & 25.89 & Linoleic acid & $\mathrm{C} 18 \mathrm{H} 32 \mathrm{O} 2$ & 280.452 & 280.24 & 5.197132616 & 279.234[M-H]- & 2 \\
\hline 14 & 25.97 & Tangshenoside III (2) & $\mathrm{C} 34 \mathrm{H} 46 \mathrm{O} 17$ & 726.725 & 726.274 & 2.035763411 & $727.28[\mathrm{M}+\mathrm{H}]+$ & 1 \\
\hline 15 & 26.19 & $\begin{array}{l}\text { 5-Mpe-bis(hobz) } \\
\text { phenol }\end{array}$ & $\mathrm{C} 29 \mathrm{H} 26 \mathrm{O} 4$ & 438.523 & 438.183 & 7.101449275 & $\begin{array}{c}483.1843 \\
{[\mathrm{M}+\mathrm{CHOO}]-}\end{array}$ & 1 \\
\hline 16 & 26.26 & Cryptotanshinone (1) & $\mathrm{C} 19 \mathrm{H} 2003$ & $\mathrm{C} 19 \mathrm{H} 2003$ & 296.366 & 1.700879765 & $\begin{array}{c}341.1384 \\
{[\mathrm{M}+\mathrm{CHOO}]-}\end{array}$ & 3 \\
\hline 17 & 26.36 & Notoginsenoside T1 & $\mathrm{C} 36 \mathrm{H} 60010$ & 652.866 & 652.419 & 4.65437788 & $651.4141[\mathrm{M}-\mathrm{H}]-$ & 2 \\
\hline 18 & 26.41 & Hexadecanoic acid & $\mathrm{C} 16 \mathrm{H} 32 \mathrm{O} 2$ & 256.43 & 256.24 & 7.254901961 & $255.2344[\mathrm{M}-\mathrm{H}]-$ & $1,2,3$ \\
\hline 19 & 26.5 & Protopanaxadiol & $\mathrm{C} 30 \mathrm{H} 52 \mathrm{O} 3$ & 460.743 & 460.392 & 3.436853002 & 483.38[M+Na]+ & 2 \\
\hline 20 & 26.77 & Lutein & $\mathrm{C} 4 \mathrm{OH} 56 \mathrm{O} 2$ & 568.886 & 568.428 & 4.323001631 & $\begin{array}{c}613.4233 \\
{[\mathrm{M}+\mathrm{CHOO}]-}\end{array}$ & 2 \\
\hline 21 & 26.86 & Ginsenoside Rh4 (6) & $\mathrm{C} 36 \mathrm{H} 6008$ & 620.868 & 620.429 & 0.080775444 & $\begin{array}{c}619.4213[\mathrm{M}-\mathrm{H}]- \\
665.4235 \\
{[\mathrm{M}+\mathrm{CHOO}]-}\end{array}$ & 2 \\
\hline 22 & 26.98 & Sanchinoside B1 (5) & $\mathrm{C} 36 \mathrm{H} 62 \mathrm{O} 9$ & 638.883 & 638.439 & 3.328100471 & $\begin{array}{c}637.4297[\mathrm{M}-\mathrm{H}]- \\
683.4306 \\
{[\mathrm{M}+\mathrm{CHOO}]-}\end{array}$ & 2 \\
\hline 23 & 27.04 & Dicaffeoyl phthalate & $\mathrm{C} 24 \mathrm{H} 38 \mathrm{O} 4$ & 390.564 & 390.277 & 5.907928389 & $\begin{array}{c}391.2827[\mathrm{M}+\mathrm{H}]+ \\
413.2671[\mathrm{M}+\mathrm{Na}]+\end{array}$ & 1,2 \\
\hline 24 & 29.2 & Gypenoside XVII & $\mathrm{C} 48 \mathrm{H} 82 \mathrm{O} 18$ & 947.166 & 946.55 & 2.237687366 & 933.5447[M-H]- & 2 \\
\hline 25 & 29.3 & Gypenosideix & $\mathrm{C} 47 \mathrm{H} 80017$ & 917.14 & 916.54 & 4.901960784 & $917.5432[\mathrm{M}+\mathrm{H}]+$ & 2 \\
\hline
\end{tabular}

${ }^{a}$ 1: Codonopsis pilosula (Franch.) Nannf.; 2: Panax notoginseng (Burkill) F.H.Chen; 3: Nardostachys jatamansi (D.Don) DC.; 4: Polygonatum kingianum Collett \& Hemsl. The eight components in italic are common components in vivo and in vitro. Reference: Wang and Qian (2010). 


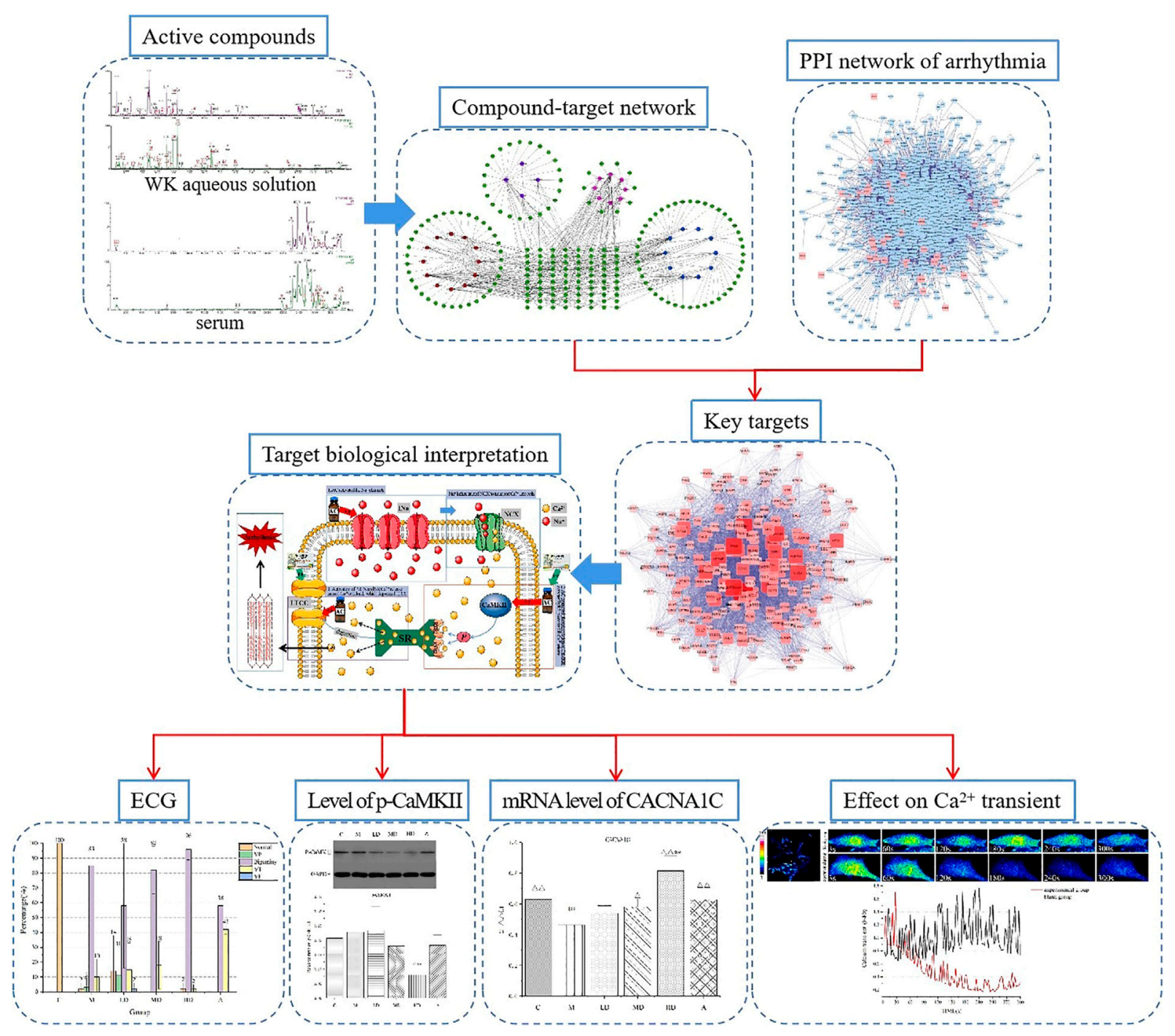

FIGURE 1 | Flowchart for the systems pharmacology approach used in this study.

\section{Identification of Key Targets}

Analysis of the common targets of WK and arrhythmia revealed 33 key targets (Table 3). These targets were regarded to be the key targets for WK-mediated treatment of arrhythmia.

\section{Pathway Construction and Analysis}

The pathway information of the 33 key targets revealed 75 KEGG pathways, including the calcium, oxytocin, adrenergic signaling in cardiomyocytes, adipocytokine, and dilated cardiomyopathy signaling pathways. The pathways were closely associated with arrhythmias (Supplementary Appendix S3) based on their $p$-values. Figure 4 highlights the first 20 pathways obtained by DAVID enrichment. In addition, the calcium signaling pathway was involved in 34 pathways and indirectly related to 33 pathways. Therefore, we presumed that the calcium signaling pathway is closely related to arrhythmia. The CaMKII, CNCA1C, and $\mathrm{Ca}^{2+}$ of the calcium signaling pathway were thus selected as the observation index in this study based on these findings combined with other relevant literature.

Calcium regulation plays a central role in cardiac function. The precise handling of cytoplasmic calcium concentration during the excitation-contraction coupling (ECC) is an essential aspect of arrhythmia pathophysiology. The L-type $\mathrm{Ca}^{2+}$ current (LTCC) plays a crucial role in the ECC. In the same line, membrane depolarization in ventricular myocytes during the action potential (AP) causes synchronous activation of multiple LTCCs, consequently triggering the release of $\mathrm{Ca}^{2+}$ from the sarcoplasmic reticulum (SR). These occurrences increase the intracellular $\mathrm{Ca}^{2+}$ that initiates contraction. $\mathrm{Ca}^{2+}$ influx via the LTCC provides a multifunctional signal that triggers muscle contraction, controls AP, and regulates gene expression (Shaw and Colecraft, 2013). As such, a dysregulation of the calcium channel function may lead to cardiac rhythm disorders. Cav1.2 is encoded by the Voltage-dependent L-type 


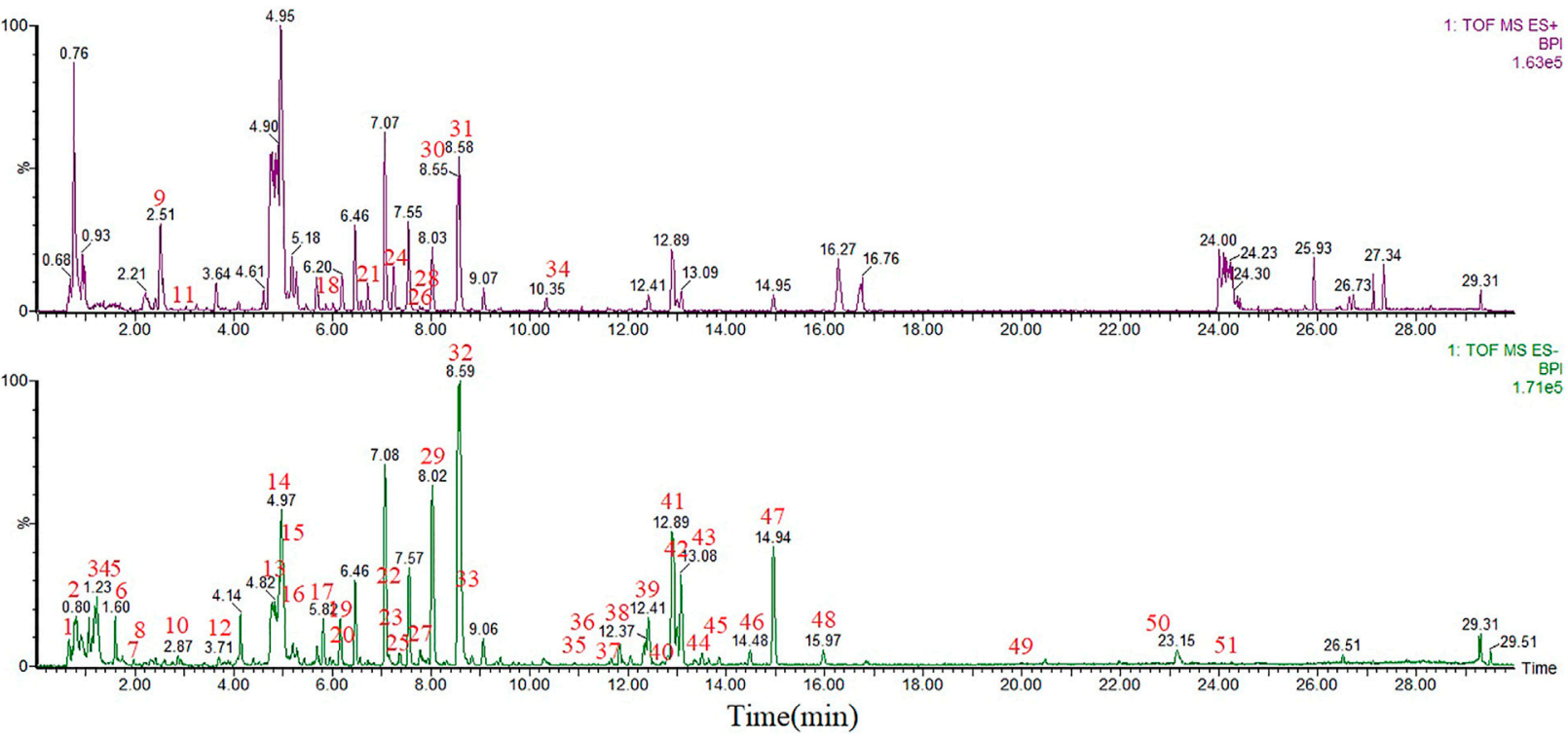

FIGURE 2 | UPLC-MS base peak intensity chromatograms of WK aqueous solution in negative and positive modes.
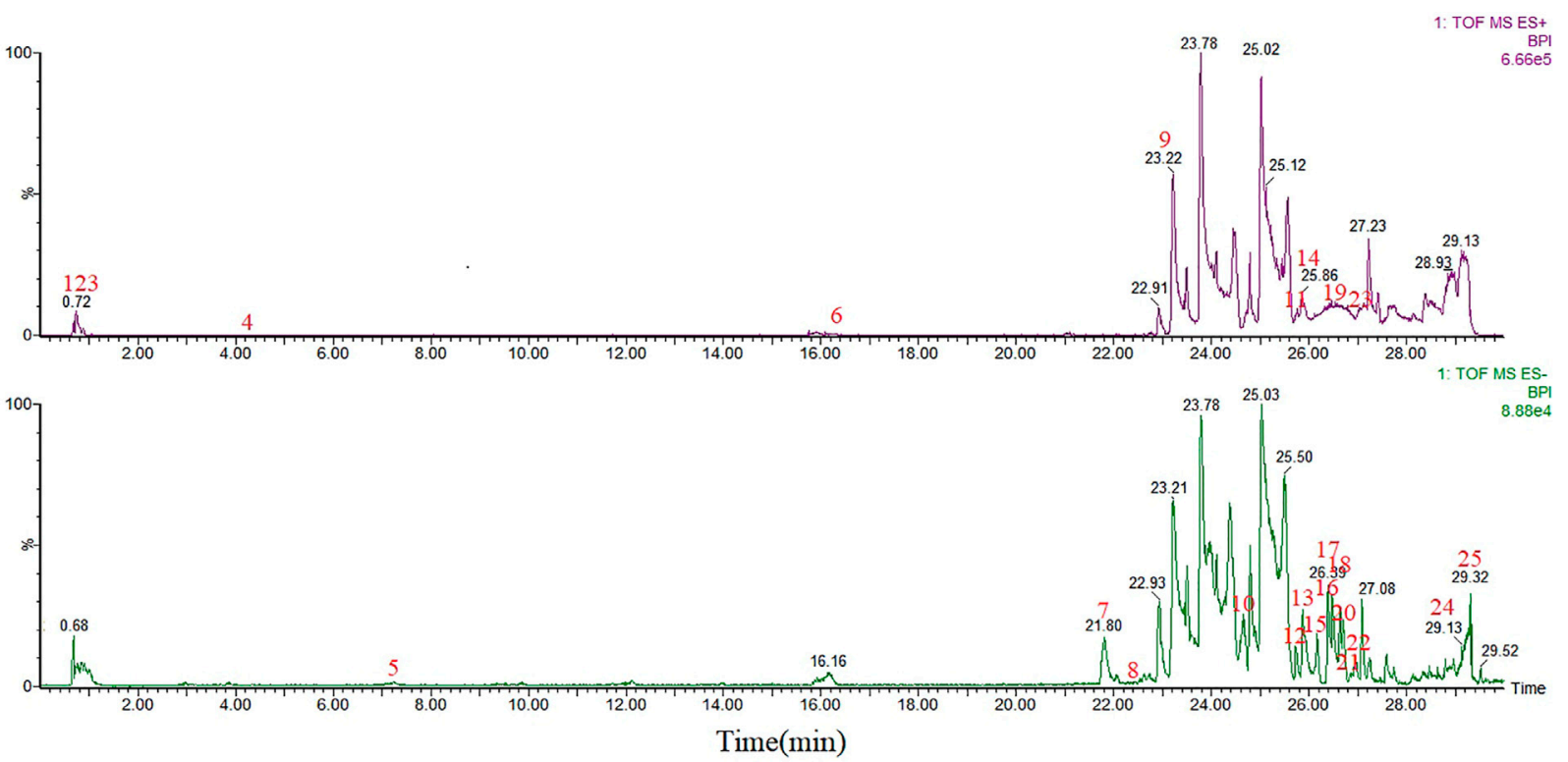

FIGURE 3 | UPLC-MS base peak intensity chromatograms of serum after WK administration in negative and positive modes.

TABLE 3 | Anti-arrhythmic targets of WK (gene name).

\begin{tabular}{|c|c|c|c|c|c|c|c|c|c|c|c|}
\hline No. & Key target & No. & Key target & No. & Key target & No. & Key target & No. & Key target & No. & Key target \\
\hline 1 & ADRA1D & 7 & BCL2L1 & 13 & HSP90AB1 & 19 & NFKB1 & 25 & PLG & 31 & SOD1 \\
\hline 2 & ADRA2A & 8 & CASP3 & 14 & JUN & 20 & NOS1 & 26 & PRKACA & 32 & TNF \\
\hline 3 & ADRB1 & 9 & CDKN1A & 15 & $\mathrm{KCNH} 2$ & 21 & NOS2 & 27 & PTEN & 33 & TP53 \\
\hline 4 & AHSA1 & 10 & EGFR & 16 & LYZ & 22 & NR3C1 & 28 & RELA & & \\
\hline 5 & $\mathrm{AR}$ & 11 & ESR1 & 17 & $\mathrm{MDH} 2$ & 23 & OPRD1 & 29 & SCN5A & & \\
\hline 6 & BAX & 12 & FOS & 18 & MYC & 24 & PFKM & 30 & SLC6A3 & & \\
\hline
\end{tabular}




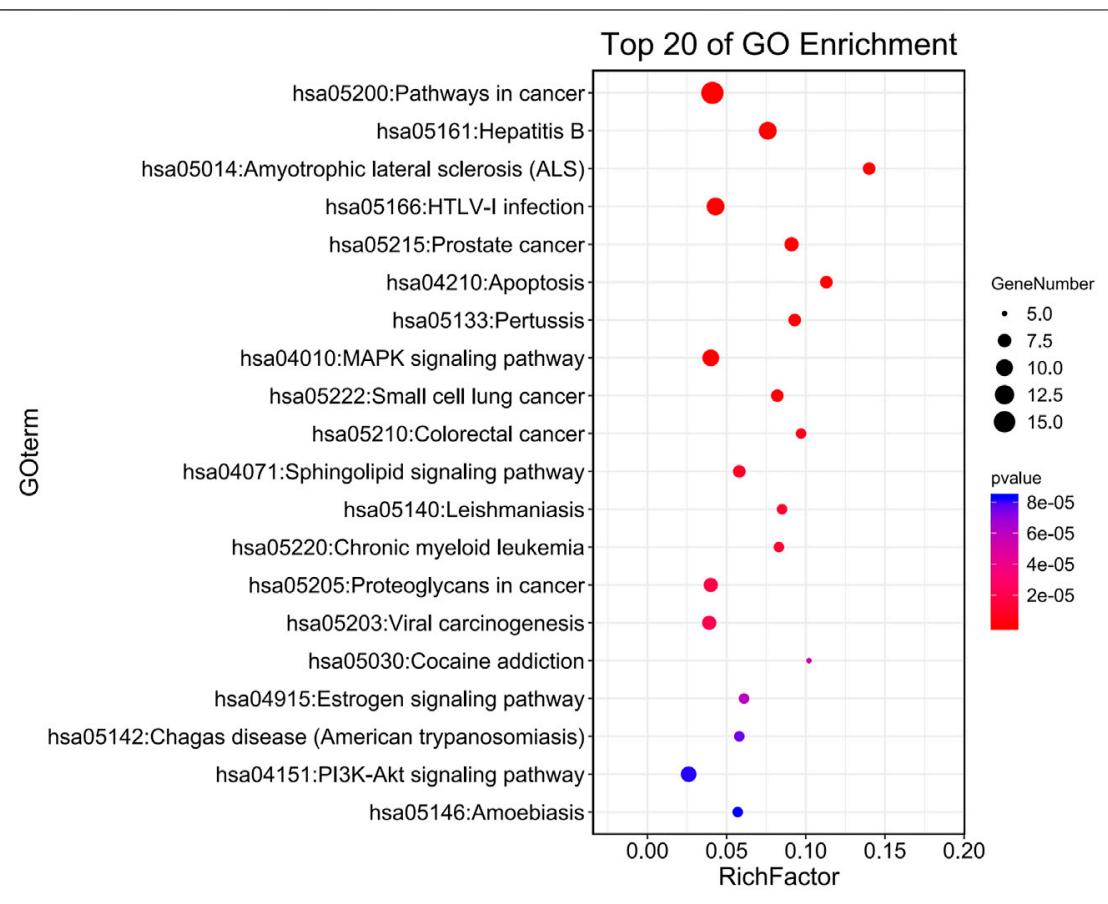

FIGURE 4 |KEGG pathway analysis. The larger RichFactor is, the greater the degree of enrichment is; The range of $p$ value is $[0,1]$, and the closer it is to 0 , the more significant the enrichment is.

calcium channel subunit alpha-1C (CACNA1C) and regulated by the calcium/calmodulin-dependent protein kinaseII (CaMKII). Thus, an increase in the expression and activation of CaMKII causes an increase in CaV1.2 phosphorylation and diastolic SR $\mathrm{Ca}^{2+}$ leakage (Ai et al., 2005). The diastolic SR Ca ${ }^{2+}$ leak in return initiates early afterdepolarizations (EADs) (Wu et al., 1999) and delayed afterdepolarizations (DADs) (Xing et al., 2013).

Therefore, we hypothesized that WK could inhibit arrhythmias by regulating the CaMKII/CACNA1C/Ca ${ }^{2+}$ pathway. The assumption is that WK suppresses CaMKII phosphorylation and CACNA1C activation and antagonizes the intracellular $\mathrm{Ca}^{2+}$ overload to avoid the occurrence of EADs and DADs. CaMKII, CACNA1C, and $\mathrm{Ca}^{2+}$ concentration were thus selected as the observation indices of this study.

\section{Wenxin Keli Significantly Reduced AC-Induced Arrhythmia}

1) Mortality: There were no deaths in groups $C, H D$, and $A$ during the experiments. However, two animals in group $\mathrm{M}$ and one animal in the groups LD and MD died. The animals died of ventricular fibrillation (VF) after pleomorphic ventricular tachycardia (Polymorphic VT). Nonetheless, the ECG recording time of the dead rats was very short and thus could not be compared with that of other rats. As such, we only analyzed the ECG of the surviving rats. All groups had different degrees of arrhythmia except group C.

2) The initial time and duration of arrhythmias: There were insignificant differences in the initial time and duration of arrhythmias among the five experimental groups $(p>0.05$;
Table 4). The average initial time and duration were 615.26 and $3,901.07 \mathrm{~s}$, respectively. However, there were significant differences in arrhythmia percentage between the groups $(p<$ 0.01 ; Figure 5). The arrhythmia order was ventricular premature (VP), bigeminy, VT, and VF based on severity. Notably, rats in group HD were mainly characterized by bigeminy (96\%), with low proportions of VT and no VP during the onset of arrhythmia. The group exhibited the best inhibitory effect on tachycardia.

3) Types of arrhythmias: VT and bigeminy were observed in the five experimental groups. However, VP was observed in groups $M, L D$, and $A$, normal ECG was observed in groups $M, L D$, and $\mathrm{HD}$, while VF was observed in group LD (Figure 5).

4) ECG parameters: Compared with ECG parameters before the experiment, R-R, $\mathrm{P}-\mathrm{R}$, and $\mathrm{Q}-\mathrm{T}$ interval did not change in groups $\mathrm{C}$ and $\mathrm{M}$. However, they were prolonged in groups $\mathrm{LD}$, $\mathrm{MD}$, and $\mathrm{A}$, and shortened in group HD (Table 5). In the same line, APD was lengthened in groups $\mathrm{LD}, \mathrm{MD}$, and $\mathrm{A}$, especially group Abut shortened in group HD. These findings suggested that high-dose WK played an antiarrhythmic role by shortening APD.

\section{Wenxin Keli Significantly Inhibits p-CaMKII}

Group M had increased p-CaMKIl levels than group C, suggesting that AC could activate CaMKII to induce arrhythmia. In the same line, the expression of p-CaMKII decreased in all groups except group LD after WK and amiodarone treatment, with group HD having the most significant decrease $(p<0.05)$. Notably, group HD had a significantly lower level of p-CaMKII than group C $(p<$ 0.01 ), suggesting that a high WK dose markedly inhibited the expression of CaMKII (Table 6; Figure 6). 
TABLE 4 | The initial time and duration of arrhythmias $(\bar{x} \pm s, n=8)$.

\begin{tabular}{|c|c|c|c|c|c|c|}
\hline \multirow[t]{2}{*}{ Group } & \multirow[t]{2}{*}{ Control } & \multirow[t]{2}{*}{ Model } & \multicolumn{3}{|c|}{ WK } & \multirow[t]{2}{*}{ Amiodarone } \\
\hline & & & Low dose & Medium dose & High dose & \\
\hline Initial time(s) & None & $534.67 \pm 69.95$ & $569.33 \pm 40.61$ & $696 \pm 351.24$ & $687 \pm 109.16$ & $589.33 \pm 296.38$ \\
\hline \multicolumn{7}{|l|}{ Duration(s) } \\
\hline Total & 1200 & $2,834.67 \pm 420.39$ & $2,619 \pm 337.76$ & $6,459 \pm 5,264.6$ & $2,342 \pm 230.65$ & $4,506 \pm 791.57$ \\
\hline Bigeminy & 0 & $2,227.5 \pm 507.01$ & $1,583 \pm 828.46$ & $5,071.33 \pm 3,571.4$ & $2,301.67 \pm 235.92$ & $3,133.75 \pm 1146.31$ \\
\hline $\mathrm{VT}$ & 0 & $413 \pm 383.25$ & $732.5 \pm 406.59$ & $2,081.5 \pm 2,069.7$ & $121 \pm 0$ & $1,929.33 \pm 462.48$ \\
\hline VP & 0 & 346 & 1450 & 0 & 0 & 254 \\
\hline VF & 0 & 0 & 193 & 0 & 0 & 0 \\
\hline Normal & 1,200 & $964 \pm 1,028.13$ & 1827 & 0 & 170 & 0 \\
\hline
\end{tabular}

$V T$, ventricular tachycardia; VP, ventricular premature; VF, ventricular fibrillation.
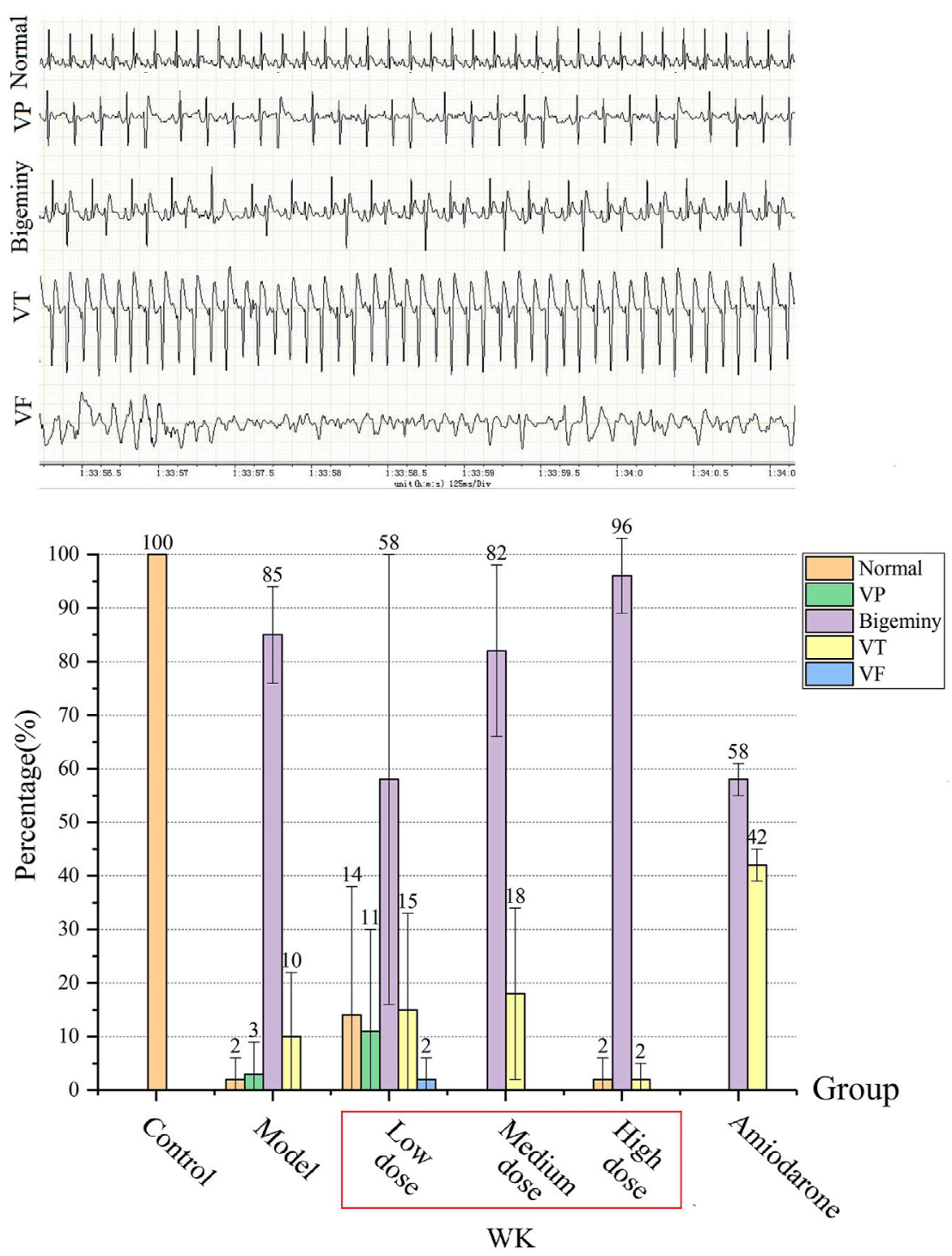

FIGURE 5 | The percentage of arrhythmias' duration $(\%)(\bar{x} \pm s, n=8)$. VP: ventricular premature, VT: ventricular tachycardia, VF: ventricular fibrillation. 
TABLE 5 | ECG parameters under the influence of WK $(\bar{x} \pm s, n=8)$.

\begin{tabular}{|c|c|c|c|c|c|c|}
\hline \multirow[t]{2}{*}{ Group } & \multicolumn{3}{|c|}{ Before } & \multicolumn{3}{|c|}{ After } \\
\hline & RR (ms) & PR (ms) & QT (ms) & RR (ms) & PR (ms) & QT (ms) \\
\hline Control & $132 \pm 4.47$ & $43.4 \pm 1.34$ & $55.2 \pm 1.48$ & $132 \pm 4.47$ & $43.4 \pm 1.34$ & $55.2 \pm 1.48$ \\
\hline Model & $132 \pm 4.47$ & $45 \pm 1.41$ & $55 \pm 1$ & $132 \pm 4.47$ & $45 \pm 1.41$ & $55 \pm 1$ \\
\hline \multicolumn{7}{|l|}{ WK } \\
\hline Low dose & $128 \pm 4.47$ & $44 \pm 1.58$ & $55 \pm 1$ & $138 \pm 4.47^{\star \star}$ & $49.2 \pm 1.3^{\star \star}$ & $60.4 \pm 0.89^{\star \star}$ \\
\hline Medium dose & $132 \pm 4.47$ & $44.6 \pm 1.52$ & $54.6 \pm 1.95$ & $142 \pm 4.47^{\star \star}$ & $50.2 \pm 1.3^{\star \star}$ & $61 \pm 1.58^{\star \star}$ \\
\hline High dose & $134 \pm 5.48$ & $44.2 \pm 1.92$ & $53.6 \pm 2.79$ & $124 \pm 5.48^{\star}$ & $39.4 \pm 1.95^{\star \star}$ & $49 \pm 2.55^{\star}$ \\
\hline Amiodarone & $132 \pm 4.47$ & $45 \pm 2$ & $54.2 \pm 1.3$ & $162 \pm 4.47^{\star \star}$ & $60 \pm 1.58^{\star \star}$ & $70 \pm 1.58^{\star \star}$ \\
\hline
\end{tabular}

${ }^{*} \mathrm{p}<0.05,{ }^{* *} \mathrm{p}<0.01$, versus before experiment.

\begin{tabular}{lc}
\hline TABLE 6 | The level of P-CaMK $\|$ protein $(\bar{x} \pm s, n=8)$. & \\
\hline Group & p-CaMK II \\
\hline Control & $0.21 \pm 0.03$ \\
Model & $0.23 \pm 0.04$ \\
WK & \\
Low dose & $0.23 \pm 0.08$ \\
Medium dose & $0.18 \pm 0.09$ \\
High dose & $0.08 \pm 0.03^{\triangle \triangle} \triangle \triangle_{\star *}$ \\
Amiodarone & $0.18 \pm 0.04$
\end{tabular}

${ }^{*} \mathrm{p}<0.05,{ }^{* *} \mathrm{p}<0.01$, versus the control group. ${ }^{{ }^{*}} \mathrm{p}<0.05, \Delta_{\mathrm{p}}<0.01$, versus the model group.

\begin{tabular}{lc}
\hline TABLE $7 \mid$ The level of CACNA1C $(\bar{x} \pm s, n=8)$. & \\
\hline Group & CACNA1C \\
\hline Control & $0.63 \pm 0.05^{\Delta \Delta}$ \\
Model & $0.46 \pm 0.08^{\star \star}$ \\
WK & \\
Low dose & $0.54 \pm 0.05$ \\
Medium dose & $0.58 \pm 0.05^{\triangle}$ \\
High dose & $0.81 \pm 0.1^{\triangle \triangle \star \star}$ \\
Amiodarone & $0.63 \pm 0.07^{\triangle \Delta}$
\end{tabular}

${ }^{*} \mathrm{p}<0.05,{ }^{* *} \mathrm{p}<0.01$, versus the control group. ${ }^{{ }} \mathrm{p}<0.05,{ }^{\Delta} \mathrm{p}<0.01$, versus the model group.
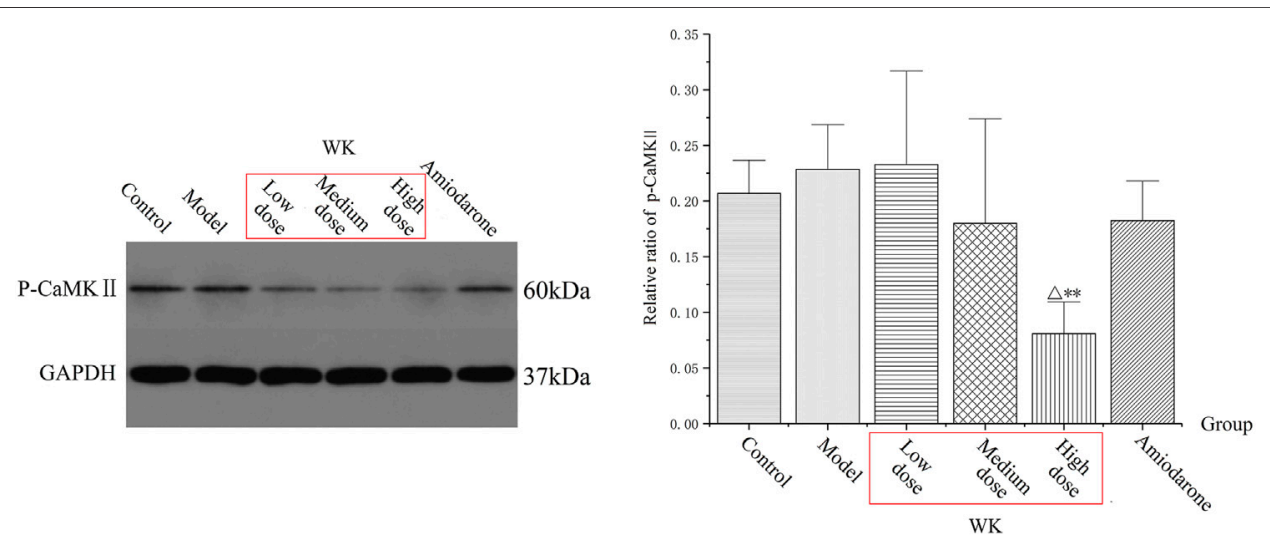

FIGURE 6 | The levels of $p$-CaMKII protein. $(\bar{x} \pm s, n=8){ }^{\star} p<0.05$, ${ }^{\star \star} p<0.01$, versus the control group. ${ }^{\Delta} p<0.05,{ }^{\Delta} p<0.01$, versus the model group.

\section{Wenxin Keli Significantly Increased CACNA1C}

Group M had decreased CACNA1C levels $(p<0.01)$ than group C, suggesting that $\mathrm{AC}$ could inhibit CACNA1C to induce arrhythmia. Compared with group $\mathrm{M}$, the expression of CACNA1C increased in all groups except group LD after treatment of WK and amiodarone, with group HD exhibiting the most significant increase $(p<0.01)$. Notably, group HD had a significantly higher level of CACNA1C than group C $(p<0.01)$, suggesting that a high WK dose markedly increased the expression of CACNA1C (Table 7; Figure 7).

\section{Wenxin Keli Significantly Reduced the Concentration of Intracellular $\mathbf{C a}^{2+}$}

Cytoplasmic $\mathrm{Ca}^{2+}$ transients were determined in the experimental and blank groups (high dose of WK) to evaluate the time-course effects of WK on intracellular $\mathrm{Ca}^{2+}$ homeostasis. WK significantly reduced the resting $\mathrm{Ca}^{2+}$ ratio and the amplitude of $\mathrm{Ca}^{2+}$ ratio within $5 \mathrm{~min}$ after adding WK $(p<0.01)$. WK also induced a decrease in the cytoplasmic $\mathrm{Ca}^{2+}$ ratio within $5 \mathrm{~min}$ in a time-dependent manner (Figure 8; Supplementary Appendix S4). These results indicated that inhibition of $\mathrm{Ca}^{2+}$ overload is potentially an essential mechanism of the WK-induced antiarrhythmic effect. 


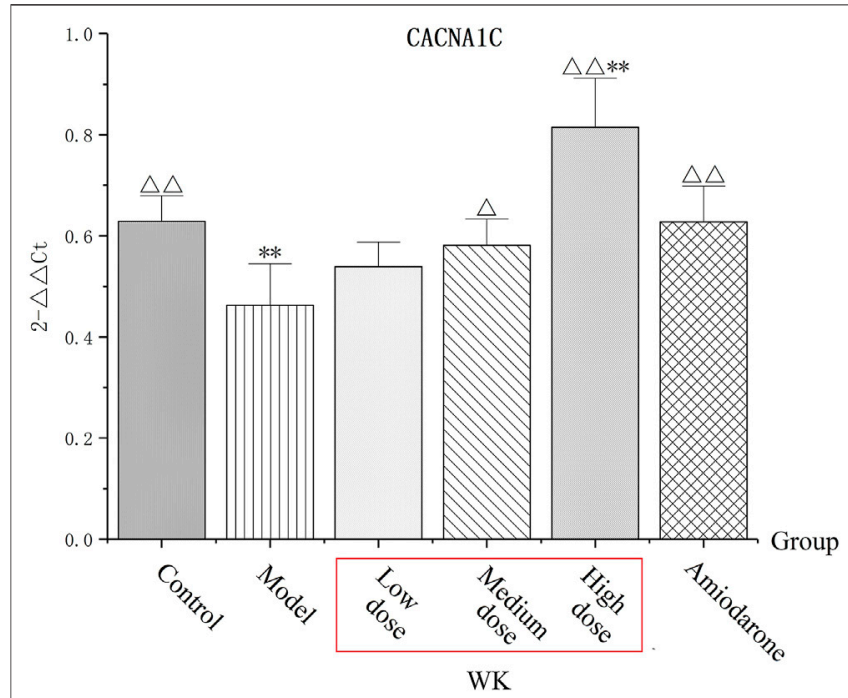

FIGURE 7 | The level of CACNA1C. $(\bar{x} \pm s, n=8){ }^{\star} p<0.05,{ }^{* \star} p<0.01$, versus the control group. ${ }^{\Delta} p<0.05,{ }^{\Delta} p p<0.01$, versus the model group.

\section{DISCUSSION}

\section{Systems Pharmacology Approach Used in Traditional Chinese Medicine Research}

To date, the action mechanisms of TCM remain to be completely understood despite TCM having been known since ancient times to have therapeutic effects on human cardiovascular diseases (Jauhari et al., 2016). The drug discovery and development process are time-consuming, expensive, and challenging. The systems pharmacology approach can assist in shortening this time and potentially reduce the cost of drug research and development. In this paper, the pharmacology approach was used to predict the potential targets and action pathways of WK in arrhythmias treatment. Moreover, the study combined UPLC/Q-TOF-MS and animal-based experiments to identify WK's active compounds and action mechanisms for arrhythmias treatment.

\section{Mechanism of AC-Induced Arrhythmias}

AC is a major bioactive diterpenoid alkaloid derived from aconitum plants. It has been used in the past as an antipyretic, analgesic, antirheumatic drug, and a neurotransmission inhibitor (Herzog et al., 1964; Sato et al., 1979; Hikino et al., 1980; Fraser et al., 2003). However, it is toxic to the heart and the central nervous system (Honerjäger and Meissner, 1983; Catterall et al., 1992; Ameri, 1998). Arrhythmias are a major side effect of AC (Zhu et al., 2017), including the induction of VT, torsades de pointes, and VF (Lu and De Clerck, 1993). AC-induced arrhythmias are a consequence of a combination of multiple mechanisms. Studies postulate that AC increases the excitability of ectopic rhythms (Qiu et al., 2016), decreases the APA of papillary muscle APs, shortens the APD90 and APD30 (Chen et al., 2018), and leads to tachyarrhythmias.

1) Promote $\mathrm{Na}^{+}$Influx: $\mathrm{AC}$ acts as an $\mathrm{INa}$ agonist that opens the $\mathrm{Na}^{+}$channels during the depolarization/repolarization phases. This occurrence suppresses the conformational change of $\mathrm{Na}^{+}$ channels from the active to the inactive state, causing the membrane to remain depolarized (Wright, 2002; Wang and Wang, 2003). Large $\mathrm{Na}^{+}$influx into the cytosol induces

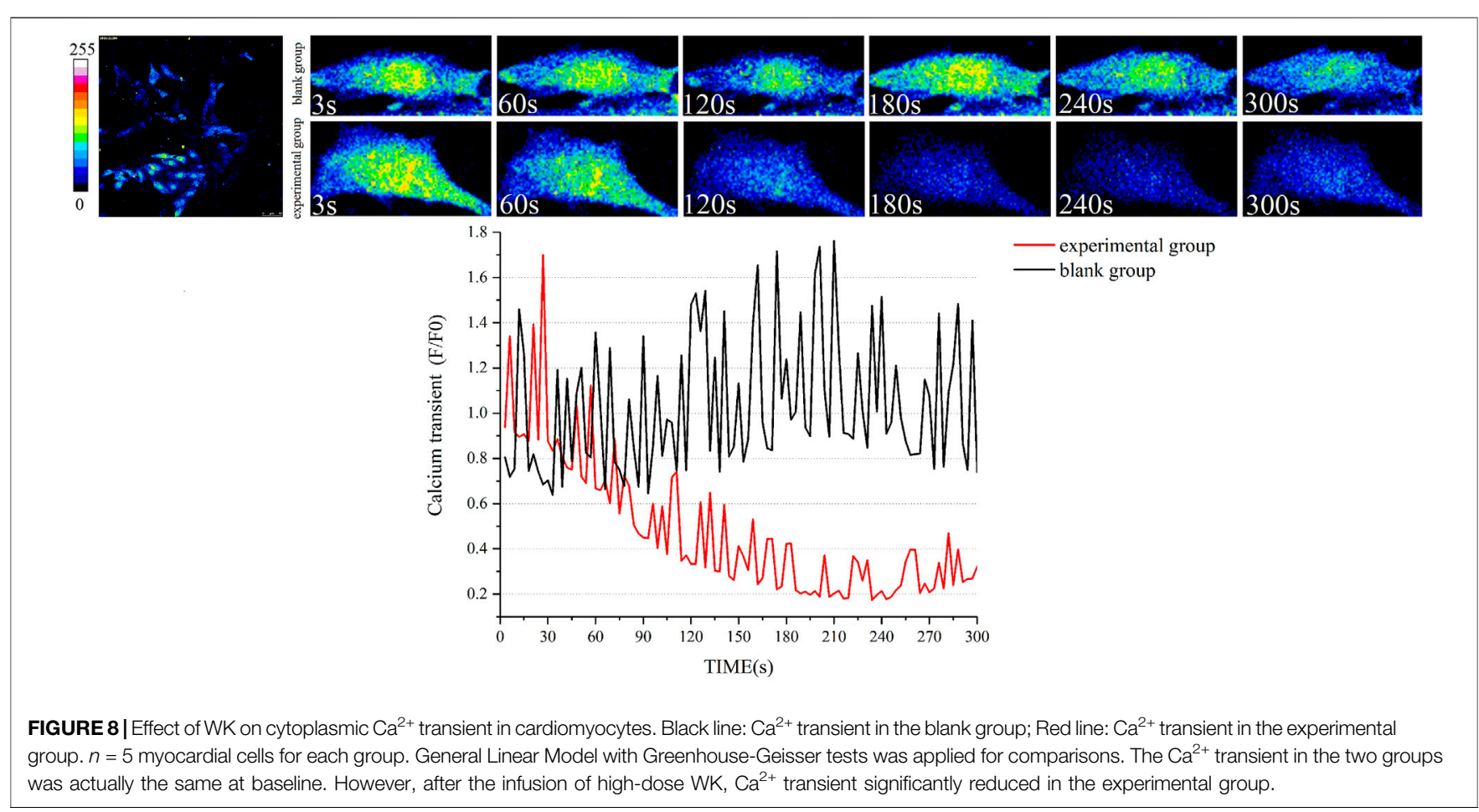


triggered activities (TA) (Sawanobori et al., 1987; Watano et al., 1999), thereby causing single or multifocal ectopic rhythms (Suzuki et al., 2014).

2) Lead to $\mathrm{Ca}^{2+}$ Overload: Abundant $\mathrm{Na}^{+}$influx into the cytosol causes $\mathrm{Ca}^{2+}$ overload via sequential activation of electrogenic $\mathrm{Na}^{+}-\mathrm{Ca}^{2+}$ exchanger (NCX), thus inducing DAD and TA (Wang et al., 2008; Zhou et al., 2013). As a result, $\mathrm{Ca}^{2+}$ channel antagonists, such as verapamil, exhibit better therapeutic effects on AC-induced VTs than $\mathrm{Na}^{+}$channel antagonists, such as quinidine (Takahara et al., 1999; Van Landeghem et al., 2007) in clinical practice.

3) Inhibition of Outward $\mathrm{K}^{+}$Currents: AC significantly increases $\mathrm{Ca}^{2+}$ influx, inhibits outward $\mathrm{K}^{+}$currents, prolongs repolarization, and produces $\mathrm{DAD}$ and reentry, resulting in arrhythmias (Mitamura et al., 2002).

4) Inhibition of LTCC: LTCC is activated upon membrane depolarization and $\mathrm{Ca}^{2+}$ influx. It triggers the release of $\mathrm{Ca}^{2+}$ via the $\mathrm{Ca}^{2+}$ release channels, ryanodine receptors (RyRs) of the SR. During normal AP, early LTCC peaks trigger robust SR release followed by partial inactivation because of two processes: $\mathrm{Ca}^{2+}$-and voltage-dependent inactivation. LTCC regulates AP repolarization and is vital in developing cardiac arrhythmias such as VF, the leading cause of sudden cardiac death (Moroz and Lipnitskii, 2006; Kranias and Bers, 2007). Studies postulate that AC-induced inhibitions of LTCC are vital in the proarrhythmic effects of AC in humans. AC can block LTCC following a mechanism independent from increased INa (Fu et al., 2007). The effects of $\mathrm{AC}$ on cardiac repolarization and beating frequency in hiPSC-CMs resemble that of nifedipine (Wu et al., 2017). Moreover, inactivating LTCC channels potentially contributes to intracellular $\mathrm{Ca}^{2+}$ elevation (Ferreira et al., 1997). In contrast, some studies suggest that AC accelerates the activation of LTCC and delays the inactivation of LTCC. These studies further suggest that AC increases $\mathrm{Ca}^{2+}$ influx through LTCC (Zhou et al., 2013). These data inconsistencies are attributed to the differences in the two types of myocytes because ionic channel characters of neonatal and adult ventricular myocytes are not completely identical. In this study, the expression of CACNA1C, the gene that encodes LTCC, was significantly inhibited by AC.

5) Others: AC also stimulates the vagus nerve and inhibits the sinoatrial node and conduction system, resulting in a slowed heart rate and conduction block (Jung et al., 2011; Ono et al., 2013). Moreover, the cardiac toxicity of AC is associated with an increase in free radicals caused by oxidative stress (Wang et al., 2008). AC cardiotoxicity is also attributed to $\mathrm{Ca}^{2+}$ overload and apoptosis via the p38 signaling pathway (Sun et al., 2014).

In the present study, the antagonistic effects of WK on ACinduced arrhythmia were observed in rats. ECG recording experiments demonstrated that AC administration decreased the heart rate and prolonged the Q-T intervals, leading to VP, coupled rhythm, and tachycardia. These occurrences ultimately induced VF and rat mortality. These findings are consistent with those of Qiu et al. (2016). In contrast, pre-administration of WK effectively delayed the onset of ventricular premature beat and coupled rhythm, thereby prolonging the survival time of rats.

\section{The Role of $\mathrm{Ca}^{2+}$ in Maintaining the Heart Rhythm}

Cardiac contraction and relaxation are mediated by a precise and coordinated linkage of electrical activation (excitation) and intracellular $\mathrm{Ca}^{2+}$ homeostasis, resulting in excitationcontraction coupling. Ventricular AP starts with a sodium $\left(\mathrm{Na}^{+}\right)$influx through voltage-gated $\mathrm{Na}^{+}$channels that depolarize the cell. Voltage-sensitive LTCC is activated at a certain threshold, allowing the influence of $\mathrm{Ca}^{2+}$ into the cytosol, which triggers a much larger $\mathrm{Ca}^{2+}$ release from the $\mathrm{SR}$, the main intracellular $\mathrm{Ca}^{2+}$ storage organelle (Bers, 2002; Gambardella et al., 2018). Notably, sporadic subcellular localized $\mathrm{Ca}^{2+}$ releases are observed in myocardial cells during diastole as spatiotemporally restricted $\mathrm{Ca}^{2+}$ sparks (Cheng and Lederer, 2008). They originate from a single ryanodine receptor type 2 (RyR2) or a RyR2s cluster and represent the elementary events of cardiac ECC. The recruitment and summation of many $\mathrm{Ca}^{2+}$ sparks at the beginning of systole produces abundant whole-cell $\mathrm{Ca}^{2+}$ transients. The process is referred to as $\mathrm{Ca}^{2+}$-induced $\mathrm{Ca}^{2+}$ release (CICR) and is the fundamental link between electrical and mechanical activation in the heart (Fabiato, 1983). The cytosolic $\mathrm{Ca}^{2+}$ then binds to troponin $\mathrm{C}$ and initiates myocardial contractions to form the cardiac systole. In contrast, myofilaments relax during diastole. The relaxation is caused by $\mathrm{Ca}^{2+}$ re-uptake into the SR by the SR $\mathrm{Ca}^{2+}$-ATPase type-2a (SERCA2a) that pumps $\mathrm{Ca}^{2+}$ back into the SR stores. It is also caused by $\mathrm{Ca}^{2+}$ extrusion and releases into the extracellular space through NCX, which exchanges three $\mathrm{Na}^{+}$ions entering for one $\mathrm{Ca}^{2+}$ ion leaving the cell (Stern and Cheng, 2004). The NCX removes $\mathrm{Ca}^{2+}$ by generating a net inward depolarizing current, called the transient inward current (Iti). SERCA2a takes up approximately $63 \%$ of cytosolic $\mathrm{Ca}^{2+}$, while NCX extrudes $37 \%$ of Ca2+ in humans (Bassani et al., 1994; Terentyev et al., 2002). The depolarization and release of $\mathrm{Ca}^{2+}$ into the cytosol and its subsequent rapid re-uptake or extrusion results in a $\mathrm{Ca}^{2+}$ wave referred to as the $\mathrm{Ca}^{2+}$ transient. The amount of $\mathrm{Ca}^{2+}$ released from the SR directly correlates with the $\mathrm{Ca}^{2+}$ transient amplitude. It is responsible for the strength of systolic contraction. Notably, any alterations in intracellular $\mathrm{Ca}^{2+}$ handling results in electrical stability and cardiac contractility changes, leading to malignant ventricular arrhythmias. Principally, $\mathrm{Ca}^{2+}$ overload is an important AC mechanism that causes arrhythmia. Analyses of $\mathrm{Ca}^{2+}$-signaling parameters in cultured myocardial cells are traditionally based on the use of ratiometric $\mathrm{Ca}^{2+}$ dyes such as Fluo- 4 followed by detection of spatiotemporal changes in their fluorescence intensities using epifluorescence or confocal microscopy (Whitaker, 2010; Prasad and Inesi, 2012). In this protocol, we describe an experimental approach suitable for evaluating $\mathrm{Ca}^{2+}$ signals in myocardial cells. This approach revealed that WK significantly reduces intracellular $\mathrm{Ca}^{2+}$ transients, thus highlighting it as a potential mechanism by which WK treats arrhythmias. 


\section{The Role of Calcium/ Calmodulin-Dependent Protein Kinasell in Antiarrhythmia}

During the initial stage of AP, $\mathrm{Ca}^{2+}$ flowing into LTCC through mycoplasma voltage gating triggers $\mathrm{SR}$ to release large amounts of $\mathrm{Ca}^{2+}$. This myocardial contraction and blood drawing process driven by $\mathrm{Ca}^{2+}$ is called ECC (Winslow et al., 2016). In the same line, CaMKII is a key downstream regulator in $\mathrm{Ca}^{2+}$ related physiological activities, such as autophosphorylation and posttranslational modification. It also plays an important role in the excitation-contraction coupling and relaxation events of cardiomyocytes (Jiang and Wang, 2020). CaMKII belongs to the subfamily of multifunctional Ser/Thr kinases, which phosphorylate various substrates and regulate numerous cellular functions (Soderling, 1999; Fujisawa, 2001; Hook and Means, 2001; Rusciano et al., 2012) intimately involved in heart diseases (Braun and Schulman, 1995; Erickson et al., 2008; Anderson et al., 2011). It catalyzes the phosphorylation of $\beta$ Thr498 (Grueter et al., 2006), additional sites on the CaV1.2 a-subunit (Lee et al., 2006; Hudmon et al., 2019), phospholamban (Bilezikjian et al., 1981), and ryanodine receptors (Wehrens et al., 2004). CaMKII mediated phosphorylation of the ryanodine receptor increases the open probability of sarcoplasmic $\mathrm{Ca}^{2+}$ load. In the same line, phosphorylation of the LTCC leads to a slower inactivation, while phosphorylation of phospholamban leads to an increase in sarcoplasmic $\mathrm{Ca}^{2+}$ load (Anderson, 2005; Guo and Duff, 2006). Consequently, these actions cause an up-regulated CaMKII activity during cardiac pathology, leading to an increased intracellular $\mathrm{Ca}^{2+}$ concentration. This increase leads to triggered activity via spontaneous diastolic $\mathrm{Ca}^{2+}$ release (Sossalla et al., 2010). Studies also postulate that acute CaMKII inhibition approaches are antiarrhythmic in numerous animal studies (Mazur et al., 1999; Gbadebo et al., 2002; Bourgonje et al., 2012; Driessen et al., 2014; Takanari et al., 2016; He et al., 2019). In this study, the level of p-CaMKII in the model group was higher than that of the control group, suggesting that $\mathrm{AC}$ activated CaMKII to induce arrhythmia. AC inhibits the expression of CACNA1C, thereby inhibiting $\mathrm{Ca}^{2+}$ from entering cardiomyocytes through LTCC. Moreover, SR $\mathrm{Ca}^{2+}$ release is essential in activating CaMKII, which subsequently induces phosphorylation of RyRs and promotes the release of $\mathrm{Ca}^{2+}$ from SR. Our data showed that WK can inhibit the expression of CaMKII, suggesting it was one of the mechanisms by which WK treats arrhythmias.

\section{Fascinating L-Type $\mathrm{Ca}^{2+}$ Current}

The precise handling of cytoplasmic $\mathrm{Ca}^{2+}$ concentration during ECC is an important aspect of arrhythmia pathophysiology. ECC is an essential effector of the LTCC Cav1.2, the main isoform expressed in ventricular cardiomyocytes (Bers, 2002). The L-type refers to "long-lasting," a historic nomenclature that antedates molecular identification of the determinants of ICa. ICa is a slow inward current that contrasts the more rapid kinetics of the $\mathrm{Na}^{+}$ current. Cav1.2 is a pore-forming a-subunit of LTCCs, indicating that it is a member of the voltage-gated ( $\mathrm{v}) \mathrm{Ca}^{2+}$ channel family. It is structurally homologous to voltage-gated $\mathrm{Na}^{+}$channels and belongs to a family of six membrane-spanning voltage-gated ion channels. CACNA1C, CACNB2, and CACNA2D1 genes encode the pore-forming a-subunit (Cav1.2) and the $\mathrm{b} 2$ and a2d ancillary subunits that form the cardiac $\mathrm{Ca}^{2+}$ channel, which generates the LTCC (Rougier and Abriel, 2016). LTCCs are distributed in small clusters of about 10-12 channels along the sarcolemma of these cells (Bers and Stiffel, 1993; Franzini-Armstrong et al., 1999; Soeller et al., 2007; Dixon et al., 2015). LTCCs open to allow $\mathrm{Ca}^{2+}$ to enter the cell once the membrane potential is reached during the plateau phase of the ventricular AP. The $\mathrm{Ca}^{2+}$ signal is amplified via $\mathrm{Ca}^{2+}$-induced $\mathrm{Ca}^{2+}$ release by opening $\mathrm{RyRs}$ from the SR, causing a cell-wide increase of $\mathrm{Ca}^{2+}$, which triggers cell contraction (Cheng et al., 1993; Cheng et al., 1996). ICa is a critical determinant of intracellular $\mathrm{Ca}^{2+}$ transients that trigger transmitter release, secretion, and contraction (Catterall, 2000). The size of the intracellular $\mathrm{Ca}^{2+}$ transients in the heart is determined by the release of $\mathrm{Ca}^{2+}$ from intracellular stores and the size of the LTCC (Bers, 2002). LTCC is an essential source of inward current (ICa) for prolonging APD (Alseikhan et al., 2002). CaV1.2 proteins are subject to multiple modes of regulation that determine the number of available channels by trafficking or modulating channel activation or inactivation via protein kinases and accessory proteins. As such, numerous variables determine LTCC activity. The human CACNA1C gene located on chromosome 12 encodes for the pore-forming CaV1.2 subunit protein of the cardiac LTCC (Powers et al., 1991; Soldatov et al., 1998). Abnormal expression of CACNA1C thus leads to LTCC dysfunction.

ICaL must inhibit the transportation of $\mathrm{Ca}^{2+}$ out of the cytosol primarily via the sarcolemmal NCX and the SR $\mathrm{Ca}^{2+}$-ATPase (SERCA) for relaxation to occur in normal conditions. This phenomenon consequently takes $\mathrm{Ca}^{2+}$ back into the SR. NCX operates in both the $\mathrm{Ca}^{2+}$ efflux and influx (or reverse) modes, depending on the internal and external concentrations of $\mathrm{Na}^{+}$and $\mathrm{Ca}^{2+}$. INa is elevated in AC-induced myocytes, while NCX works almost exclusively in the $\mathrm{Na}^{+}$extrusion mode, thus significantly increasing the amount of $\mathrm{Ca}^{2+}$ influx. This influx raises the cellular and $\mathrm{SR} \mathrm{Ca}^{2+}$ content, resulting in larger $\mathrm{Ca}^{2+}$ transients. In contrast, other studies postulate that $\mathrm{SR} \mathrm{Ca}^{2+}$ release modulates the sarcolemmal ICaL, suggesting a retrograde in communication between the $\mathrm{SR}$ and the sarcolemmal LTCC in cardiac ECC (Balog and Gallant, 1999). Moreover, AC -induces SR $\mathrm{Ca}^{2+}$ leakage through the RyR2 channel, thereby depressing ICaL. These reports support the retrograde hypothesis in communication between the SR $\mathrm{Ca}^{2+}$ release channel and the LTCC (CACNA1C) (Fu et al., 2008). Notably, this communication may be mediated by a direct interaction between the two-channel proteins, LTCC and RyR2, in some cases (Nakai et al., 1996). Studies postulate that AC decreased the expression level of SERCA but increase that of NCX (Zhou et al., 2013).

This study showed that AC has a significant inhibitory effect on LTCC, indicating that AC-induced intracellular $\mathrm{Ca}^{2+}$ overload is not achieved by increasing LTCC. However, these findings contrasted those of Souza et al. (2019) and Koenig et al. (2014), who reported that AC increased LTCC expression. Notably, the 


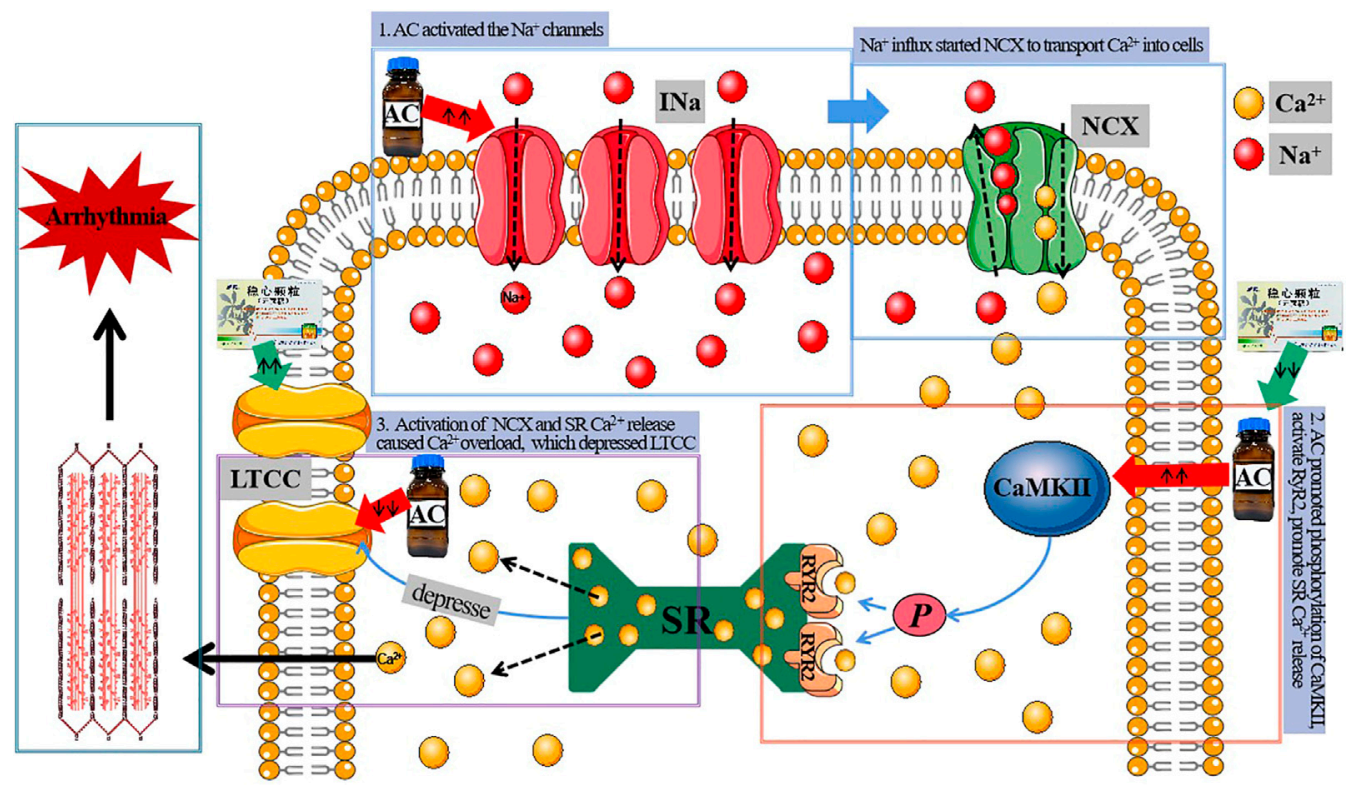

FIGURE 9 | Mechanism of treating arrhythmia with WK.

studies report the existence of NCX and a retrograde in communication between the $\mathrm{SR} \mathrm{Ca}^{2+}$ release channel and LTCC. In this study, WK significantly reduced intracellular $\mathrm{Ca}^{2+}$ transients, thus confirming that the increased expression of NCX and the presence of $\mathrm{Ca}^{2+}$ influx mode were the primary causes of AC-induced arrhythmia. AC also increased the expression of $\mathrm{p}$-CaMKIl, thus promoting the release of $\mathrm{Ca}^{2+}$ from SR. The $\mathrm{Ca}^{2+}$ release of SR inhibited LTCC because of the retrograde communication between them. QRT-PCR results of CACNA1C also confirmed these findings. Similarly, laserscanning confocal calcium imaging directly demonstrated the inhibitory effect of WK on intracellular $\mathrm{Ca}^{2+}$ concentration. Maintaining intracellular $\mathrm{Ca}^{2+}$ balance may thus be the primary mechanism of WK against arrhythmia.

\section{Highlights and Innovation of the Pharmacology Approach}

1) It is a good model for TCM research: The approach also detected the chemical components of WK besides the antiarrhythmia mechanism and correlated the potential active components with their pharmacodynamic mechanisms. The research model was more systematic and complete, thus proving a good model for TCM research.

2) It provides a research direction of antiarrhythmic effects of TCM: Besides the most relevant calcium signaling pathway, other signaling pathways were also detected, thus providing a good direction for subsequent studies. The pathways included the oxytocin signaling pathway, adrenergic signaling in cardiomyocytes, adipocytokine signaling pathway, and dilated cardiomyopathy associated with the metabolism of adrenaline, angiotensin, fats, glycometabolism, apoptotic factors, and other arrhythmia-related factors. These findings demonstrate that TCM treatment of diseases has a multi-target comprehensive effect, thus further proving that systems pharmacology is an effective method in TCM study.

3) Construction of appropriate disease model: The present study used AC to construct a pure arrhythmia rat model instead of the toxic arrhythmia that causes myocardial infarction and heart failure. The pure arrhythmia model was more conductive to reveal the pathogenesis of arrhythmia because it did not interfere with the complex pathological mechanisms.

4) New antiarrhythmic strategy: This study demonstrated that WK had therapeutic effects in AC-induced rat arrhythmias by inhibiting $\mathrm{p}$-CaMKIl, over-expressing $\mathrm{CNCA1C}$, and inhibiting intracellular $\mathrm{Ca}^{2+}$ transients. CaMKII and NCX play a vital role in $\mathrm{Ca}^{2+}$ balance. As such, blocking CaMKII and NCX can be a safe and effective strategy against arrhythmias.

\section{CONCLUSION}

\section{Antiarrhythmic Potential Active Components and Targets of Wenxin Keli}

UPLC/Q-TOF-MS identified 68 components both in vivo and in vitro as the potential active components of WK. Among them, 33 key targets were screened based on their corresponding targets of WK potential active ingredients and targets of arrhythmia. These targets were regarded as the antiarrhythmic targets of WK (Figure 1). This study proposes that the calcium signaling pathway is the primary signaling pathway targeted by WK in arrhythmia treatment because of the WK targets, including CaMKII, CNCA1C, and $\mathrm{Ca}^{2+}$, involved in the calcium signaling pathway. It supposes that WK inhibits arrhythmia by regulating CaMKII, CNCA1C, and intracellular $\mathrm{Ca}^{2+}$ transients. 


\section{Mechanism of Treating Arrhythmia Using Wenxin Keli}

$\mathrm{Ca}^{2+}$ is both a charge carrier and a second messenger. As such, slight alterations in $\mathrm{Ca}^{2+}$ concentration during ECC significantly impacts arrhythmia vulnerability (Eisner et al., 2017). AC activates the $\mathrm{Na}^{+}$channels, thus causing a substantial $\mathrm{Na}^{+}$ influx into the cytosol, which induces TA. In the same line, NCX transport $\mathrm{Ca}^{2+}$ into cells to reduce the $\mathrm{Na}^{+}$content. AC also promotes the phosphorylation of CaMKII, activates RyR2, and promotes $\mathrm{SR} \mathrm{Ca}^{2+}$ release. Intracellular $\mathrm{Ca}^{2+}$ markedly increases and induces $\mathrm{Ca}^{2+}$ overload, consequently increasing the frequency of propagating $\mathrm{Ca}^{2+}$ waves and subsequent escalation of the propensity for triggered arrhythmia (Tiso et al., 2001; Wang et al., 2008). The intracellular $\mathrm{Ca}^{2+}$ overload triggered by $\mathrm{Ca}^{2+}$ signals is a potential mechanism of AC-induced cardiac arrhythmia (Zhou et al., 2013). There is a retrograde communication between the SR $\mathrm{Ca}^{2+}$ release channel and the LTCC (CNCA1C). Moreover, AC-induced SR $\mathrm{Ca}^{2+}$ leakage through the RyR2 channel further inhibits LTCC (Figure 9).

However, pretreatment with WK lowers mortality, lessens malignant arrhythmias, and shortens RR, PR, and QT intervals than AC-induced rats. WK induces some antiarrhythmic effects by inhibiting p-CaMKII and intracellular $\mathrm{Ca}^{2+}$ transients and overexpressing CNCA1C, thus suppressing $\mathrm{SR} \mathrm{Ca}^{2+}$ release, which is the most probable mechanism of WK inhibition of the $\mathrm{Ca}^{2+}$ overload. Maintaining intracellular $\mathrm{Ca}^{2+}$ balance is, therefore, an essential mechanism of WK against arrhythmia. The antiarrhythmic mechanism of WK also suggests that TCM has a multi-target comprehensive effect against diseases. Notably, high dose WK has the best impact and should thus be highly considered during clinical treatment.

\section{Tips for Antiarrhythmic Targets}

CaMKII activity reflects the frequency of cytosolic $\mathrm{Ca}^{2+}$ oscillations. As such, its activation is directly linked to increased APD, EADs, and arrhythmias (Mazur et al., 1999). The $\mathrm{Ca}^{2+}$ homeostatic proteins involved in E-C are CaMKII targets (Couchonnal and Anderson, 2008). APD prolongation because of increased ICaL requires $\mathrm{SR} \mathrm{Ca}^{2+}$ to activate CaMKII, which then binds to and phosphorylates $\beta 2 \mathrm{a}$ to trigger EADs. Therefore, CaMKII can serve as a novel therapeutic target for arrhythmias.

Similarly, NCX blocking is a promising safe and effective strategy against repolarization-dependent arrhythmias because

\section{REFERENCES}

Ai, X., Curran, J. W., Shannon, T. R., Bers, D. M., and Pogwizd, S. M. (2005). Ca 2+/ Calmodulin-dependent Protein Kinase Modulates Cardiac Ryanodine Receptor Phosphorylation and Sarcoplasmic Reticulum Ca2+ Leak in Heart Failure. Circ. Res. 97, 1314-1322. doi:10.1161/01.RES.0000194329.41863.89

Alseikhan, B. A., DeMaria, C. D., Colecraft, H. M., and Yue, D. T. (2002). Engineered Calmodulins Reveal the Unexpected eminence of Ca2+ Channel Inactivation in Controlling Heart Excitation. Proc. Natl. Acad. Sci. 99, 17185-17190. doi:10.1073/pnas.262372999

Ameri, A. (1998). The Effects of Aconitum Alkaloids on the central Nervous System. Prog. Neurobiol. 56, 211-235. doi:10.1016/s0301-0082(98)00037-9 it causes intracellular $\mathrm{Na}^{+}$accumulation and reversal, leading to a high cytoplasmic $\mathrm{Ca}^{2+}$ influx.

\section{DATA AVAILABILITY STATEMENT}

The original contributions presented in the study are included in the article/Supplementary Material. Further inquiries can be directed to the corresponding authors.

\section{ETHICS STATEMENT}

The animal study was reviewed and approved by the Ethics Committee for the Use of Experimental Animals at the Institute of Radiation Medicine Chinese Academy of Medical Sciences (Approval No.: IRM-DWLL-2018077).

\section{AUTHOR CONTRIBUTIONS}

All authors listed have made a substantial, direct, and intellectual contribution to the work and approved it for publication.

\section{FUNDING}

The present study was supported by the Natural Science Foundation of China (grant no. 82004329).

\section{ACKNOWLEDGMENTS}

We would like to thank HZ (from the Department of Aeronautics and Astronautics, Tsinghua University), Manager Bo Zhou (from Shandong Buchang Pharmaceutical Co., Ltd), and Manager Yujun Yan (from the Institute of Radiation Medicine Chinese Academy of Medical Sciences) for providing general support.

\section{SUPPLEMENTARY MATERIAL}

The Supplementary Material for this article can be found online at https://www.frontiersin.org/articles/10.3389/fphar.2021.704622/ full\#supplementary-material

Anderson, M. (2005). Calmodulin Kinase Signaling in Heart: an Intriguing Candidate Target for Therapy of Myocardial Dysfunction and Arrhythmias. Pharmacol. Ther. 106, 39-55. doi:10.1016/j.pharmthera.2004.11.002

Anderson, M. E., Brown, J. H., and Bers, D. M. (2011). CaMKII in Myocardial Hypertrophy and Heart Failure. J. Mol. Cell Cardiol. 51, 468-473. doi:10.1016/j.yjmcc.2011.01.012

Balog, E. M., and Gallant, E. M. (1999). Modulation of the Sarcolemmal L-type Current by Alteration in SR Ca2+ Release. Am. J. Physiology-Cell Physiol. 276, C128-C135. doi:10.1152/ajpcell.1999.276.1.C128

Bassani, J. W., Bassani, R. A., and Bers, D. M. (1994). Relaxation in Rabbit and Rat Cardiac Cells: Species-dependent Differences in Cellular Mechanisms. J. Physiol. 476, 279-293. doi:10.1113/jphysiol.1994.sp020130

Bers, D. M. (2002). Cardiac Excitation-Contraction Coupling. Nature 415 (6868), 198-205. doi:10.1038/415198a 
Bers, D. M., and Stiffel, V. M. (1993). Ratio of Ryanodine to Dihydropyridine Receptors in Cardiac and Skeletal Muscle and Implications for E-C Coupling. Am. J. Physiology-Cell Physiol. 264, C1587-C1593. doi:10.1152/ajpcell.1993.264.6.C1587

Bilezikjian, L. M., Kranias, E. G., Potter, J. D., and Schwartz, A. (1981). Studies on Phosphorylation of Canine Cardiac Sarcoplasmic Reticulum by Calmodulindependent Protein Kinase. Circ. Res. 49, 1356-1362. doi:10.1161/ 01.res.49.6.1356

Bourgonje, V. J. A., Schoenmakers, M., Beekman, J. D. M., van der Nagel, R., Houtman, M. J. C., Miedema, L. F., et al. (2012). Relevance of calmodulin/ CaMKII Activation for Arrhythmogenesis in the AV Block Dog. Heart Rhythm 9, 1875-1883. doi:10.1016/j.hrthm.2012.07.023

Braun, A. P., and Schulman, H. (1995). The Multifunctional Calcium/calmodulindependent Protein Kinase: from Form to Function. Annu. Rev. Physiol. 57, 417-445. doi:10.1146/annurev.ph.57.030195.002221

Burashnikov, A., Petroski, A., Hu, D., Barajas-Martinez, H., and Antzelevitch, C. (2012). Atrial-selective Inhibition of Sodium-Channel Current by Wenxin Keli Is Effective in Suppressing Atrial Fibrillation. Heart Rhythm 9, 125-131. doi:10.1016/j.hrthm.2011.08.027

Camm, A. J. (2017). Hopes and Disappointments with Antiarrhythmic Drugs. Int. J. Cardiol. 237, 71-74. doi:10.1016/j.ijcard.2017.03.056

Catterall, W. A., Trainer, V., and Baden, D. G. (1992). Molecular Properties of the Sodium Channel: a Receptor for Multiple Neurotoxins. Bull. Soc. Pathol. Exot. $85,481-485$

Catterall, W. A. (2000). Structure and Regulation of Voltage-Gated Ca2+ Channels. Annu. Rev. Cel Dev. Biol. 16, 521-555. doi:10.1146/annurev.cellbio.16.1.521

Chen, X., Guo, H., Li, Q., Zhang, Y., Liu, H., Zhang, X., et al. (2018). Protective Effect of Berberine on Aconite-induced M-yocardial I-njury and the A-ssociated M-echanisms. Mol. Med. Rep. 18, 4468-4476. doi:10.3892/ mmr.2018.9476

Cheng, H., Lederer, M. R., Xiao, R.-P., Gómez, A. M., Zhou, Y.-Y., Ziman, B., et al. (1996). Excitation-contraction Coupling in Heart: New Insights from Ca2+ sparks. Cell Calcium 20, 129-140. doi:10.1016/s0143-4160(96)90102-5

Cheng, H., Lederer, W., and Cannell, M. (1993). Calcium sparks: Elementary Events Underlying Excitation-Contraction Coupling in Heart Muscle. Science 262 (5134), 740-744. doi:10.1126/science.8235594

Cheng, H., and Lederer, W. J. (2008). Calcium sparks. Physiol. Rev. 88, 1491-1545. doi:10.1152/physrev.00030.2007

Couchonnal, L. F., and Anderson, M. E. (2008). The Role of Calmodulin Kinase II in Myocardial Physiology and Disease. Physiology 23, 151-159. doi:10.1152/ physiol.00043.2007

Dixon, R. E., Moreno, C. M., Yuan, C., Opitz-Araya, X., Binder, M. D., Navedo, M. F., et al. (2015). Graded Ca2+/calmodulin-dependent Coupling of VoltageGated CaV1.2 Channels. Elife 4, e05608. doi:10.7554/eLife.05608

Driessen, H. E., Bourgonje, V. J. A., van Veen, T. A. B., and Vos, M. A. (2014). New Antiarrhythmic Targets to Control Intracellular Calcium Handling. Neth. Heart J. 22, 198-213. doi:10.1007/s12471-014-0549-5

Eisner, D. A., Caldwell, J. L., Kistamás, K., and Trafford, A. W. (2017). Calcium and Excitation-Contraction Coupling in the Heart. Circ. Res. 121, 181-195. doi:10.1161/CIRCRESAHA.117.310230

Erickson, J. R., Joiner, M.-1. A., Guan, X., Kutschke, W., Yang, J., Oddis, C. V., et al. (2008). A Dynamic Pathway for Calcium-independent Activation of CaMKII by Methionine Oxidation. Cell 133, 462-474. doi:10.1016/j.cell.2008.02.048

Fabiato, A. (1983). Calcium-induced Release of Calcium from the Cardiac Sarcoplasmic Reticulum. Am. J. Physiology-Cell Physiol. 245, C1-C14. doi:10.1152/ajpcell.1983.245.1.C1

Ferreira, G., Yi, J., Ríos, E., and Shirokov, R. (1997). Ion-dependent Inactivation of Barium Current through L-type Calcium Channels. J. Gen. Physiol. 109, 449-461. doi:10.1085/jgp.109.4.449

Franzini-Armstrong, C., Protasi, F., and Ramesh, V. (1999). Shape, Size, and Distribution of Ca2+ Release Units and Couplons in Skeletal and Cardiac Muscles. Biophysical J. 77, 1528-1539. doi:10.1016/S0006-3495(99)77000-1

Fraser, S. P., Salvador, V., Manning, E. A., Mizal, J., Altun, S., Raza, M., et al. (2003). Contribution of Functional Voltage-Gated Na+ Channel Expression to Cell Behaviors Involved in the Metastatic cascade in Rat Prostate Cancer: I. Lateral Motility. J. Cel. Physiol. 195, 479-487. doi:10.1002/jcp.10312

Frommeyer, G., and Eckardt, L. (2016). Drug-induced Proarrhythmia: Risk Factors and Electrophysiological Mechanisms. Nat. Rev. Cardiol. 13, 36-47. doi:10.1038/nrcardio.2015.110
Fu, M., Li, R.-X., Fan, L., He, G.-W., Thornburg, K. L., and Wang, Z. (2008). Sarcoplasmic Reticulum Ca2+ Release Channel Ryanodine Receptor (RyR2) Plays a Crucial Role in Aconitine-Induced Arrhythmias. Biochem. Pharmacol. 75, 2147-2156. doi:10.1016/j.bcp.2008.02.027

Fu, M., Wu, M., Wang, J.-F., Qiao, Y.-J., and Wang, Z. (2007). Disruption of the Intracellular $\mathrm{Ca} 2+$ Homeostasis in the Cardiac Excitation-Contraction Coupling Is a Crucial Mechanism of Arrhythmic Toxicity in AconitineInduced Cardiomyocytes. Biochem. Biophysical Res. Commun. 354, 929-936. doi:10.1016/j.bbrc.2007.01.082

Fujisawa, H. (2001). Regulation of the Activities of Multifunctional Ca2+/ Calmodulin-dependent Protein Kinases. J. Biochem. 129, 193-199. doi:10.1093/oxfordjournals.jbchem.a002843

Gambardella, J., Trimarco, B., Iaccarino, G., and Santulli, G. (2017). New Insights in Cardiac Calcium Handling and Excitation-Contraction Coupling. Adv. Exp. Med. Biol. 1067, 373-385. doi:10.1007/5584_2017_106

Gbadebo, T. D., Trimble, R. W., Khoo, M. S. C., Temple, J., Roden, D. M., and Anderson, M. E. (2002). Calmodulin inhibitor W-7 unmasks a novel electrocardiographic parameter that predicts initiation of torsade de pointes. Circulation 105, 770-774. doi:10.1161/hc0602.103724

Grueter, C. E., Abiria, S. A., Dzhura, I., Wu, Y., Ham, A.-J. L., Mohler, P. J., et al. (2006). RETRACTED: L-type Ca2+ Channel Facilitation Mediated by Phosphorylation of the $\beta$ Subunit by CaMKII. Mol. Cel 23, 641-650. doi:10.1016/j.molcel.2006.07.006

Guo, J., and Duff, H. J. (2006). Calmodulin Kinase II Accelerates L-type Ca2+ current Recovery from Inactivation and Compensates for the Direct Inhibitory Effect of $[\mathrm{Ca} 2+] \mathrm{i}$ in Rat Ventricular Myocytes. J. Physiol. 574, 509-518. doi:10.1113/jphysiol.2006.109199

Hao, D. C., and Xiao, P. G. (2014). Network Pharmacology: a Rosetta Stone for Traditional Chinese Medicine. Drug Dev. Res. 75, 299-312. doi:10.1002/ddr.21214

He, Q., Cheng, J., and Wang, Y. (2019). Chronic CaMKII Inhibition Reverses Cardiac Function and Cardiac reserve in HF Mice. Life Sci. 219, 122-128. doi:10.1016/j.lfs.2019.01.010

Herzog, W. H., Feibel, R. M., and Bryant, S. H. (1964). The Effect of Aconitine on the Giant Axon of the Squid. J. Gen. Physiol. 47, 719-733. doi:10.1085/ jgp.47.4.719

Hikino, H., Konno, C., Takata, H., Yamada, Y., Yamada, C., Ohizumi, Y., et al. (1980). Antiinflammatory Principles of Aconitum Roots. J. PharmacobioDynamics 3, 514-525. doi:10.1248/bpb1978.3.514

Honerjäger, P., and Meissner, A. (1983). The Positive Inotropic Effect of Aconitine. Naunyn Schmiedebergs Arch. Pharmacol. 322, 49-58. doi:10.1007/BF00649352

Hong, B., He, J., Le, Q., Bai, K., Chen, Y., and Huang, W. (2019). Combination Formulation of Tetrodotoxin and Lidocaine as a Potential Therapy for Severe Arrhythmias. Mar. Drugs 17, 685. doi:10.3390/md17120685

Hook, S. S., and Means, A. R. (2001). Ca2+/CaM-DEPENDENTKINASES: From Activation to Function. Annu. Rev. Pharmacol. Toxicol. 41, 471-505. doi:10.1146/annurev.pharmtox.41.1.471

Huang, C., Zheng, C., Li, Y., Wang, Y., Lu, A., and Yang, L. (2014). Systems Pharmacology in Drug Discovery and Therapeutic Insight for Herbal Medicines. Brief Bioinform 15, 710-733. doi:10.1093/bib/bbt035

Hudmon, A., Schulman, H., Kim, J., Maltez, J. M., Tsien, R. W., and Pitt, G. S. (2005). CaMKII Tethers to L-type Ca2+ Channels, Establishing a Local and Dedicated Integrator of Ca2+ Signals for Facilitation. J. Cel Biol 171, 537-547. doi:10.1083/jcb.200505155

Iyengar, R., Zhao, S., Chung, S.-W., Mager, D. E., and Gallo, J. M. (2012). Merging Systems Biology with Pharmacodynamics. Sci. Translational Med. 4, $126 \mathrm{ps} 7$. doi:10.1126/scitranslmed.3003563

Jauhari, N., Gupta, S., Saxena, S., and Bharadvaja, N. (2016). Computational Studies of Synthetic and Plant-Derived Compounds against Cardiovascular Disease Targets. Ijpsdr 8, 144e148. doi:10.25004/IJPSDR.2016.080303

Jiang, S. J., and Wang, W. (2020). Research Progress on the Role of CaMKII in Heart Disease. Am. J. Transl Res. 12, 7625-7639.

Johannes, C. B., Varas-Lorenzo, C., McQuay, L. J., Midkiff, K. D., and Fife, D. (2010). Risk of Serious Ventricular Arrhythmia and Sudden Cardiac Death in a Cohort of Users of Domperidone: a Nested Case-Control Study. Pharmacoepidem. Drug Safe. 19, 881-888. doi:10.1002/pds.2016

John, R. M., Tedrow, U. B., Koplan, B. A., Albert, C. M., Epstein, L. M., Sweeney, M. O., et al. (2012). Ventricular Arrhythmias and Sudden Cardiac Death. Lancet 380, 1520-1529. doi:10.1016/S0140-6736(12)61413-5 
Jung, B.-C., Lee, S.-H., Cho, Y.-K., Park, H.-S., Kim, Y.-N., Lee, Y.-S., et al. (2011). Role of the Alternans of Action Potential Duration and Aconitine-Induced Arrhythmias in Isolated Rabbit Hearts. J. Korean Med. Sci. 26, 1576-1581. doi:10.3346/jkms.2011.26.12.1576

Koenig, X., Rubi, L., Obermair, G. J., Cervenka, R., Dang, X. B., Lukacs, P., et al. (2014). Enhanced Currents through L-type Calcium Channels in Cardiomyocytes Disturb the Electrophysiology of the Dystrophic Heart. Am. J. Physiology-Heart Circulatory Physiol. 306 (4), H564-H573. doi:10.1152/ ajpheart.00441.2013

Kranias, E. G., and Bers, D. M. (2007). Calcium and Cardiomyopathies. Subcell Biochem. 45, 523-537. doi:10.1007/978-1-4020-6191-2_20

Kuhn, M., Szklarczyk, D., Franceschini, A., von Mering, C., Jensen, L. J., and Bork, P. (2012). STITCH 3: Zooming in on Protein-Chemical Interactions. Nucleic Acids Res. 40 (Database issue), D876-D880. doi:10.1093/nar/gkr1011

Lee, T.-S., Karl, R., Moosmang, S., Lenhardt, P., Klugbauer, N., Hofmann, F., et al. (2006). Calmodulin Kinase II Is Involved in Voltage-dependent Facilitation of the L-type Cav1.2 Calcium Channel. J. Biol. Chem. 281, 25560-25567. doi:10.1074/jbc.M508661200

Li, B., Tao, W., Zheng, C., Shar, P. A., Huang, C., Fu, Y., et al. (2014). Systems Pharmacology-Based Approach for Dissecting the Addition and Subtraction Theory of Traditional Chinese Medicine: An Example Using Xiao-ChaihuDecoction and Da-Chaihu-Decoction. Comput. Biol. Med. 53, 19-29. doi:10.1016/j.compbiomed.2014.05.007

Liang, Bo., Jing, M.-T., and Liao, H.-L. (2019). Research and Prospective of Traditional Chinese Medicine on Sodium Ion Channels. Drug Combination 7erapy 1, 2-8.

Liang, Bo., Liang, W.-L., and Liao, H.-L. (2018). Efficacy and Safety of Mahuang Fuzi Xixin Decoction on Sick Sinus Syndrome: a Meta-Analysis. TMR Integr. Med. 2, 30-38.

Liu, Y. W., Guo, J. H., Zhang, P., and Li, C. (2009). The Effects of Nardostachys Chinensis Betal Extract on the Sodium Current and Transient Outward Potassium Current of Rat Ventricular Myocytes. Chin J. Card. Paceing Electrophysiol 23, 533-535.

Livak, K. J., and Schmittgen, T. D. (2001). Analysis of Relative Gene Expression Data Using Real-Time Quantitative PCR and the $2-\Delta \Delta C T$ Method. Methods 25 (4), 402-408. doi:10.1006/meth.2001.1262

Lu, H. R., and Clerck, F. D. (1993). R 56 865, a Na+/Ca2+-Overload Inhibitor, Protects against Aconitine-Induced Cardiac Arrhythmias In Vivo. J. Cardiovasc. Pharmacol. 22, 120-125. doi:10.1097/00005344-19930700000019

Luo, A., Liu, Z., Cao, Z., Hao, J., Wu, L., Fu, C., et al. (2017). Wenxin Keli Diminishes Ca2+ Overload Induced by Hypoxia/reoxygenation in Cardiomyocytes through Inhibiting INaL and ICaL. Pacing Clin. Electrophysiol. 40, 1412-1425. doi:10.1111/pace.13206

Mazur, A., Roden, D. M., and Anderson, M. E. (1999). Systemic administration of calmodulin antagonist $\mathrm{W}-7$ or protein kinase A inhibitor $\mathrm{H}-8$ prevents torsade de pointes in rabbits. Circulation 100, 2437-2442. doi:10.1161/ 01.cir.100.24.2437

McKenna, W. J., Asaad, N. A., and Jacoby, D. L. (2019). Prediction of Ventricular Arrhythmia and Sudden Death in Arrhythmogenic Right Ventricular Cardiomyopathy. Eur. Heart J. 40, 1859-1861. doi:10.1093/eurheartj/ehz195

Mitamura, M., Horie, S., Sakaguchi, M., Someya, A., Tsuchiya, S., Van de Voorde, J., et al. (2002). Mesaconitine-induced Relaxation in Rat Aorta: Involvement of $\mathrm{Ca} 2+$ Influx and Nitric-Oxide Synthase in the Endothelium. Eur. J. Pharmacol. 436, 217-225. doi:10.1016/s0014-2999(01)01623-5

Moroz, V. M., and Lipnitskii, T. N. (2006). Dysfunction of Ionic Channels in Cardiomyocyte Sarcolemma and Cardiac Arrhythmias. Bull. Exp. Biol. Med. 141, 397-399. doi:10.1007/s10517-006-0181-5

Nakai, J., Dirksen, R. T., Nguyen, H. T., Pessah, I. N., Beam, K. G., and Allen, P. D. (1996). Enhanced Dihydropyridine Receptor Channel Activity in the Presence of Ryanodine Receptor. Nature 380, 72-75. doi:10.1038/380072a0

O’Rourke, B., Liu, T., and Foster, D. B. (2016). Seeing the Forest for the Trees. Circ. Res. 119, 1170-1172. doi:10.1161/CIRCRESAHA.116.310066

Ono, T., Hayashida, M., Tezuka, A., Hayakawa, H., and Ohno, Y. (2013). Antagonistic Effects of Tetrodotoxin on Aconitine-Induced Cardiac Toxicity. J. Nippon Med. Sch. 80, 350-361. doi:10.1272/jnms.80.350

Powers, P. A., Gregg, R. G., Lalley, P. A., Liao, M., and Hogan, K. (1991). Assignment of the Human Gene for the al Subunit of the Cardiac DHP-
Sensitive Ca2+ Channel (CCHL1A1) to Chromosome 12p12-Pter. Genomics 10, 835-839. doi:10.1016/0888-7543(91)90471-p

Prasad, A. M., and Inesi, G. (2012). Analysis of Calcium Transients in Cardiac Myocytes and Assessment of the Sarcoplasmic Reticulum Ca2+-ATPase Contribution. Methods Mol. Biol. 798, 411-421. doi:10.1007/978-1-61779-343-1_24

Qiu, M., Dong, Y.-h., Han, F., Qin, J.-m., Zhang, H.-n., Du, J.-x., et al. (2016). Influence of Total Flavonoids Derived fromChoerospondias Axillaris Foliumon Aconitine-Induced Antiarrhythmic Action and Hemodynamics in Wistar Rats. J. Toxicol. Environ. Health A 79, 878-883. doi:10.1080/15287394.2016.1193117

Rougier, J.-S., and Abriel, H. (2016). Cardiac Voltage-Gated Calcium Channel Macromolecular Complexes. Biochim. Biophys. Acta (Bba) - Mol. Cel Res. 1863 , 1806-1812. doi:10.1016/j.bbamcr.2015.12.014

Rusciano, M. R., Maione, A. S., and Illario, M. (2012). Sisters Acts: Converging Signaling between CaMKII and CaMKIV, Two Members of the Same Family. Transl Med. Unisa 4, 66-72.

Salerno, D. M., Fifield, J., and Hodges, M. (1990). Antiarrhythmic Drug Therapy for Suppression of Ventricular Arrhythmia: Experience with 122 Patients Treated for Two Years. J. Clin. Pharmacol. 30, 226-234. doi:10.1002/j.15524604.1990.tb03466.x

Sato, H., Yamada, C., Konno, C., Ohizumi, Y., Endo, K., and Hikino, H. (1979). Pharmacological Actions of Aconitine Alkaloids. Tohoku J. Exp. Med. 128, 175-187. doi:10.1620/tjem.128.175

Sawanobori, T., Hirano, Y., and Hiraoka, M. (1987). Aconitine-induced Delayed Afterdepolarization in Frog Atrium and guinea Pig Papillary Muscles in the Presence of Low Concentrations of Ca2+. Jpn. J. Physiol. 37, 59-79. doi:10.2170/ jjphysiol.37.59

Shaw, R. M., and Colecraft, H. M. (2013). L-type Calcium Channel Targeting and Local Signalling in Cardiac Myocytes. Cardiovasc. Res. 98, 177-186. doi: $10.1093 / \mathrm{cvr} / \mathrm{cvt} 021$

Smoot, M. E., Ono, K., Ruscheinski, J., Wang, P.-L., and Ideker, T. (2011). Cytoscape 2.8: New Features for Data Integration and Network Visualization. Bioinformatics 27, 431-432. doi:10.1093/bioinformatics/btq675

Soderling, T. R. (1999). The Ca2+-calmodulin-dependent Protein Kinase cascade. Trends Biochem. Sci. 24, 232-236. doi:10.1016/s0968-0004(99)01383-3

Soeller, C., Crossman, D., Gilbert, R., and Cannell, M. B. (2007). Analysis of Ryanodine Receptor Clusters in Rat and Human Cardiac Myocytes. Proc. Natl. Acad. Sci. 104, 14958-14963. doi:10.1073/pnas.0703016104

Soldatov, N. M., Raudsepp, T., and Chowdhary, B. P. (1998). Repetitive Exon 45/ 46-Related Sequences of Human Ca2+ Channel a1C Subunit Gene. Hum. Hered. 48, 241-244. doi:10.1159/000022810

Sossalla, S., Fluschnik, N., Schotola, H., Ort, K. R., Neef, S., Schulte, T., et al. (2010). Inhibition of Elevated $\mathrm{Ca}$ 2+/Calmodulin-dependent Protein Kinase II Improves Contractility in Human Failing Myocardium. Circ. Res. 107, 1150-1161. doi:10.1161/circresaha.110.220418

Souza, D. S. d., Menezes-Filho, J. E. R. d., Santos-Miranda, A., Jesus, I. C. G. d., Silva Neto, J. A., Guatimosim, S., et al. (2019). Calcium Overload-Induced Arrhythmia Is Suppressed by Farnesol in Rat Heart. Eur. J. Pharmacol. 859, 172488. doi:10.1016/j.ejphar.2019.172488

Stern, M. D., and Cheng, H. (2004). Putting Out the Fire: what Terminates Calcium-Induced Calcium Release in Cardiac Muscle? Cell Calcium 35, 591-601. doi:10.1016/j.ceca.2004.01.013

Sun, G.-b., Sun, H., Meng, X.-b., Hu, J., Zhang, Q., Liu, B., et al. (2014). Aconitineinduced Ca2+ Overload Causes Arrhythmia and Triggers Apoptosis through P38 MAPK Signaling Pathway in Rats. Toxicol. Appl. Pharmacol. 279, 8-22. doi:10.1016/j.taap.2014.05.005

Suzuki, K., Matsumoto, A., Nishida, H., Reien, Y., Maruyama, H., and Nakaya, H. (2014). Termination of Aconitine-Induced Atrial Fibrillation by the KAChChannel Blocker Tertiapin: Underlying Electrophysiological Mechanism. J. Pharmacol. Sci. 125, 406-414. doi:10.1254/jphs.14023fp

Takahara, A., Uneyama, H., Sasaki, N., Ueda, H., Dohmoto, H., Shoji, M., et al. (1999). Effects of AH-1058, a New Antiarrhythmic Drug, on Experimental Arrhythmias and Cardiac Membrane Currents. J. Cardiovasc. Pharmacol. 33, 625-632. doi:10.1097/00005344-199904000-00016

Takanari, H., Bourgonje, V. J. A., Fontes, M. S. C., Raaijmakers, A. J. A., Driessen, H., Jansen, J. A., et al. (2016). Calmodulin/CaMKII Inhibition Improves Intercellular Communication and Impulse Propagation in the Heart and Is Antiarrhythmic under Conditions when Fibrosis Is Absent. Cardiovasc. Res. 111, 410-421. doi:10.1093/cvr/cvw173 
Terentyev, D., Viatchenko-Karpinski, S., Valdivia, H. H., Escobar, A. L., and Györke, S. (2002). Luminal Ca2+ Controls Termination and Refractory Behavior of Ca2+-Induced Ca2+ Release in Cardiac Myocytes. Circ. Res. 91, 414-420. doi:10.1161/01.res.0000032490.04207.bd

Tian, G., Sun, Y., Liu, S., Li, C., Chen, S., Qiu, R., et al. (2018). Therapeutic Effects of Wenxin Keli in Cardiovascular Diseases: An Experimental and Mechanism Overview. Front. Pharmacol. 9, 1005. doi:10.3389/fphar.2018.01005

Tian, M., Xiao, Y., Xue, J., Zhang, Y., Jia, Y., Luo, X., et al. (2019). The Expression of BNP, ET-1, and TGF-B1 in Myocardium of Rats with Ventricular Arrhythmias. Ijms 20, 5845. doi:10.3390/ijms20235845

Tiso, N., Stephan, D. A., Nava, A., Bagattin, A., Devaney, J. M., Stanchi, F., et al. (2001). Identification of Mutations in the Cardiac Ryanodine Receptor Gene in Families Affected with Arrhythmogenic Right Ventricular Cardiomyopathy Type 2 (ARVD2). Hum. Mol. Genet. 10, 189-194. doi:10.1093/hmg/10.3.189

Van Landeghem, A. A., De Letter, E. A., Lambert, W. E., Van Peteghem, C. H., and Piette, M. H. A. (2007). Aconitine Involvement in an Unusual Homicide Case. Int. J. Leg. Med 121, 214-219. doi:10.1007/s00414-006-0119-5

Wang, S. F., and Qian, Z. Z. (2010). Reference Handbook for Chinese Pharmacopeia (Volume I)-Modern Analysis Technology for Evaluating the Quality of Traditional Chinese Medicine. first ed. Zhejiang, China: Zhejiang University Press.

Wang, S., and Wang, G. K. (2003). Voltage-gated Sodium Channels as Primary Targets of Diverse Lipid-Soluble Neurotoxins. Cell Signal. 15, 151-159. doi:10.1016/s0898-6568(02)00085-2

Wang, X., Wang, X., Gu, Y., Wang, T., and Huang, C. (2013). Wenxin Keli Attenuates Ischemia-Induced Ventricular Arrhythmias in Rats: Involvement of L-type Calcium and Transient Outward Potassium Currents. Mol. Med. Rep. 7, 519-524. doi:10.3892/mmr.2012.1195

Wang, X., Wang, Y., Feng, X. Y., Lu, Y., Zhang, Y., Wang, W., et al. (2016). Systematic Review and Meta-Analysis of Randomized Controlled Trials on Wenxin Keli. Dddt Vol. 10, 3725-3736. doi:10.2147/DDDT.S112333

Wang, Y.-J., Chen, B.-S., Lin, M.-W., Lin, A.-A., Peng, H., Sung, R. J., et al. (2008). Time-Dependent Block of Ultrarapid-Delayed Rectifier K+ Currents by Aconitine, a Potent Cardiotoxin, in Heart-Derived H9c2 Myoblasts and in Neonatal Rat Ventricular Myocytes. Toxicol. Sci. 106, 454-463. doi:10.1093/ toxsci/kfn189

Watano, T., Harada, Y., Harada, K., and Nishimura, N. (1999). Effect of Na+/Ca2+ exchange Inhibitor, KB-R7943 on Ouabain-Induced Arrhythmias in guineapigs. Br. J. Pharmacol. 127, 1846-1850. doi:10.1038/sj.bjp.0702740

Wehrens, X. H. T., Lehnart, S. E., Reiken, S. R., and Marks, A. R. (2004). Ca2+/ Calmodulin-Dependent Protein Kinase II Phosphorylation Regulates the Cardiac Ryanodine Receptor. Circ. Res. 94, e61-70. doi:10.1161/ 01.RES.0000125626.33738.E2

Weiss, J. N., Garfinkel, A., Karagueuzian, H. S., Nguyen, T. P., Olcese, R., Chen, P.S., et al. (2015). Perspective: a Dynamics-Based Classification of Ventricular Arrhythmias. J. Mol. Cell Cardiol. 82, 136-152. doi:10.1016/j.yjmcc.2015.02.017

Whitaker, M. (2010). Genetically Encoded Probes for Measurement of Intracellular Calcium. Methods Cel Biol 99, 153-182. doi:10.1016/B978-0-12-3748416.00006-2

Winslow, R. L., Walker, M. A., and Greenstein, J. L. (2016). Modeling Calcium Regulation of Contraction, Energetics, Signaling, and
Transcription in the Cardiac Myocyte. Wires Syst. Biol. Med. 8, 37-67. doi:10.1002/wsbm.1322

Wright, S. N. (2002). Comparison of Aconitine-modified Human Heart (hH1) and Rat Skeletal $(\mu 1)$ Muscle $\mathrm{Na}+$ channels: an Important Role for External Na+ ions. J. Physiol. 538, 759-771. doi:10.1113/jphysiol.2001.012915

Wu, J., Wang, X., Chung, Y. Y., Koh, C. H., Liu, Z., Guo, H., et al. (2017). L-type Calcium Channel Inhibition Contributes to the Proarrhythmic Effects of Aconitine in Human Cardiomyocytes. PLoS One 12, e0168435. doi:10.1371/ journal.pone. 0168435

Wu, Y., MacMillan, L. B., McNeill, R. B., Colbran, R. J., and Anderson, M. E. (1999). CaM Kinase Augments Cardiac L-type Ca2+ Current: a Cellular Mechanism for Long Q-T Arrhythmias. Am. J. Physiology-Heart Circulatory Physiol. 276, H2168-H2178. doi:10.1152/ajpheart.1999.276.6.H2168

Xing, Y., Gao, Y., Chen, J., Zhu, H., Wu, A., Yang, Q., et al. (2013). Wenxin-Keli Regulates the Calcium/Calmodulin-dependent Protein Kinase II Signal Transduction Pathway and Inhibits Cardiac Arrhythmia in Rats with Myocardial Infarction. Evidence-Based Complement. Altern. Med. 2013, 1-15. doi:10.1155/2013/464508

Zhang, K., Shi, L., Tang, H.-J., Yang, L., and Yang, X.-C. (2017). Effect of Shen Song Yang Xin Capsule on Myocardial Electrophysiology of Acute Atrial Fibrillation in Canine Model. Chin. Med. J. (Engl) 130, 2513-2514. doi:10.4103/03666999.216404

Zhang, Z., Fang, Q., Du, T., Chen, G., Wang, Y., and Wang, D. W. (2020). CardiacSpecific Caveolin-3 Overexpression Prevents Post-Myocardial Infarction Ventricular Arrhythmias by Inhibiting Ryanodine Receptor-2 Hyperphosphorylation. Cardiology 145, 136-147. doi:10.1159/000505316

Zhou, Y.-h., Piao, X.-m., Liu, X., Liang, H.-h., Wang, L.-m., Xiong, X.-h., et al. (2013). Arrhythmogenesis Toxicity of Aconitine Is Related to Intracellular Ca2+ Signals. Int. J. Med. Sci. 10, 1242-1249. doi:10.7150/ijms.6541

Zhu, L., Wu, J., Zhao, M., Song, W., Qi, X., Wang, Y., et al. (2017). Mdrla Plays a Crucial Role in Regulating the Analgesic Effect and Toxicity of Aconitine by Altering its Pharmacokinetic Characteristics. Toxicol. Appl. Pharmacol. 320, 32-39. doi:10.1016/j.taap.2017.02.008

Conflict of Interest: The authors declare that the research was conducted in the absence of any commercial or financial relationships that could be construed as a potential conflict of interest.

Publisher's Note: All claims expressed in this article are solely those of the authors and do not necessarily represent those of their affiliated organizations or those of the publisher, the editors, and the reviewers. Any product that may be evaluated in this article, or claim that may be made by its manufacturer, is not guaranteed or endorsed by the publisher.

Copyright $\odot 2021 \mathrm{Li}$, Tian, Xu, Sun, Tao, Zhang, Cong, Deng, Chen, Yu, Du and Zhao. This is an open-access article distributed under the terms of the Creative Commons Attribution License (CC BY). The use, distribution or reproduction in other forums is permitted, provided the original author(s) and the copyright owner(s) are credited and that the original publication in this journal is cited, in accordance with accepted academic practice. No use, distribution or reproduction is permitted which does not comply with these terms. 